TRANSACTIONS OF THE

AMERICAN MATHEMATICAL SOCIETY

Volume 361, Number 9, September 2009, Pages 4653-4681

S 0002-9947(09)04647-9

Article electronically published on April 15, 2009

\title{
THE MILNOR FIBER CONJECTURE AND ITERATED BRANCHED CYCLIC COVERS
}

\author{
P. J. LAMBERSON
}

\begin{abstract}
In this paper we prove the Milnor Fiber Conjecture of Neumann and Wahl for a class of isolated complete intersection singularities obtained by taking iterated branched cyclic covers of the singularity link. We also show that if the Milnor Fiber Conjecture holds for a given splice diagram, then it holds for any equivalent diagram satisfying the semigroup condition. We illustrate the application of these theorems in an example and discuss the relationship of these singularities with Neumann and Wahl's Splice Type Conjecture.
\end{abstract}

\section{INTRODUCTION}

The local topology of a normal complex surface singularity is completely determined by a canonically associated 3-manifold called the link of the singularity. Michael Artin first asked the question: What analytic invariants of the singularity are determined by the topology and when? Since Artin's question, many conjectures have appeared suggesting a strong relationship between the analytic invariants of such a singularity and its topology under certain restricting assumptions on the topology of the link (e.g. [10, 11, 12, 13, 18, 19]). These conjectures overlap at varying levels of generality and provide both motivation and support for one another. Several of the conjectures have been verified for large families of singularities. Recently however, counterexamples have been discovered to many of these conjectures by Luengo-Velasco, Melle-Hernandez and Némethi [8].

Let $X$ be a complete intersection surface, given as the zero locus of $f:\left(\mathbb{C}^{n}, o\right) \rightarrow$ $\left(\mathbb{C}^{n-2}, o\right)$, with an isolated singularity at the origin $o \in \mathbb{C}^{n}$. The link $\Sigma$ of the singularity $(X, o)$ is the real three-dimensional manifold given by the intersection of $X$ with a sufficiently small sphere $S_{\epsilon}^{2 n-1}$ centered at the origin. The link is well defined for $\epsilon$ sufficiently small, and $X \cap B_{\epsilon}^{2 n}$ is homeomorphic to the cone on $\Sigma$. Thus, the link completely encodes the local topology of the singularity. The Milnor fiber for this singularity is $F:=f^{-1}(\lambda) \cap B_{\epsilon}^{2 n}$, where $\lambda$ is a general point of $\mathbb{C}^{n-2}$ sufficiently close to the origin and $B_{\epsilon}^{2 n}$ is a sufficiently small ball about the origin in $\mathbb{C}^{n}$. It is a smooth simply connected compact complex surface with boundary diffeomorphic to $\Sigma$ (see e.g. [9, 7]).

Received by the editors June 25, 2007.

2000 Mathematics Subject Classification. Primary 14B05, 32S55; Secondary 32S25, 32S50, $57 \mathrm{M} 12,57 \mathrm{~N} 10$

This work was carried out while the author was a Ph.D. student at Columbia University, supported by a graduate fellowship. Part of this research was completed while the author was supported by the Carl B. Boyer Memorial Fellowship. The author wishes to thank Walter Neumann for invaluable guidance in conducting this research.

(C)2009 American Mathematical Society Reverts to public domain 28 years from publication 
One conjecture that notably survived the counterexamples of $[8$ is the Casson Invariant Conjecture (CIC), which was one of the first such conjectures to have appeared. Formulated in 1990 by Neumann and Wahl [16], the CIC applies to normal surface singularities with link an integral homology three sphere, and says that for such a singularity the Casson invariant of the link $\lambda(\Sigma)$ equals one-eighth the signature of the Milnor fiber $\sigma(F)$ [16]. By formulas of Laufer and Durfee, $\sigma(F)$ is equivalent to classical analytic invariants of the singularity such as the Milnor number $\left(b_{2}(F)\right)$ and the geometric genus. The interest in this conjecture, as Neumann and Wahl point out, is that it "suggests that the Milnor fiber is a 'natural' 4-manifold which is attached to its boundary $\Sigma$," and that "analytic invariants like the Milnor number and geometric genus are determined by the link" 18. This is not the case for general hypersurface singularities.

Several techniques have been applied towards proving the CIC, and it has been verified in non-trivial cases [1, 3, 11, 16, 18. Computing the Casson invariant of $\Sigma$ is not challenging. In fact, the integral homology spheres that arise as singularity links are classified by splice diagrams 2 . These diagrams represent such a singularity link as being built up from well understood pieces, the Brieskorn spheres, by a topological operation called splicing. The splice decomposition of a homology sphere link is the well known Jaco-Shalen-Johannsen (JSJ) decomposition from 3manifold topology. The Casson invariant is additive under the splicing operation and known for the Brieskorn spheres, so the Casson invariant of a given homology sphere link can be easily computed from the corresponding diagram 16. On the other hand, computing the signature of $F$ is usually extremely difficult, and even in the cases where the CIC has been proven, often little is known about the topology of $F$. These proofs generally proceed by reformulating the conjecture in terms of the geometric genus of $(X, o)$, which is more easily computed from defining equations, and thus bypass the difficulty of understanding the topology of $F$ directly (e.g. [16, 18]).

In 2005 Neumann and Wahl published two papers suggesting that singularities with homology sphere link can be entirely understood from the JSJ, or splice, decomposition of the link. They extend the operation of splicing of links to a "splicing of the defining equations" [18, constructing equations for a normal surface singularity with link of a given topology. A singularity arising from this construction is called a splice type singularity, and they formulate the Splice Type Conjecture (STC): Every complete intersection surface singularity with homology sphere link is of splice type [18, 19. For splice type singularities, they also formulate the Milnor Fiber Conjecture (MFC), which gives a direct topological construction of the Milnor fiber in terms of the splice decomposition of the link [18]. The conjectured construction implies the CIC for splice type singularities [18.

In this paper we generalize Neumann and Wahl's proof of the MFC for hypersurfaces given by an equation of the form $f(x, y)+z^{n}=0$ to an infinite family of splice type complete intersection singularities obtained by taking iterated branched cyclic covers of the link. These are the only singularities for which the MFC is known to hold.

\section{BACKGROUND}

In sections 2.12.4 we recall relevant terminology and results needed in the rest of the paper. In section 2.1 we define the two main objects under study, the link and 
the Milnor fiber, and describe classical results regarding these objects. In section 2.2 we give the classification of integral homology sphere links by splice diagrams. In section 2.3 we define the splice type equations and describe the Splice Type Conjecture. In this section we also recall a few facts about semigroups. In section 2.4 we describe the Milnor Fiber Conjecture.

2.1. The link and the Milnor fiber. Let $X$ be a complete intersection surface, given as the zero locus of $f:\left(\mathbb{C}^{n}, o\right) \rightarrow\left(\mathbb{C}^{n-2}, o\right)$, with an isolated singularity at the origin $o \in \mathbb{C}^{n}$. Let $r: \mathbb{C}^{n} \rightarrow[0, \infty)$ be a real analytic function so that $r^{-1}(0)=o$. As in [7, for any set $S \subset \mathbb{C}^{n}$ let

$$
S_{r \leq \epsilon}=\{z \in S \mid r(z) \leq \epsilon\}
$$

and similarly for $S_{r>\epsilon}, S_{r<\epsilon}$ and so on. By an application of the Curve Selection Lemma (see e.g. 9]), $\left.r\right|_{X}$ has only finitely many critical values.

Proposition 2.1 ([7]). Let $\epsilon>0$ be such that $X_{r \leq \epsilon}$ is compact and $\left.r\right|_{X-o}$ has no critical value in $(0, \epsilon]$. Then $X_{r=\epsilon}$ is a compact real-analytic submanifold of $X$ and $X_{r \leq \epsilon}$ is homeomorphic to the cone on $X_{r=\epsilon}$.

The submanifold $X_{r=\epsilon}$ is called the link of the singularity. The following proposition implies that the link is well defined regardless of the choice of $r$ and $\epsilon$ subject to the assumptions of Proposition 2.1.

Proposition $2.2([7])$. Let $r, r^{\prime}: X \rightarrow[0, \infty)$ such that $r^{-1}(0)=\left(r^{\prime}\right)^{-1}(0)=o$. Let $\epsilon>0$ be such that the hypotheses of Proposition 2.1 are satisfied by $r$ and $\epsilon$. Then if $\epsilon^{\prime}>0$ is such that $X_{r^{\prime} \leq \epsilon^{\prime}} \subset X_{r<\epsilon}$, the hypotheses of Proposition 2.1 are also satisfied by $r^{\prime}$ and $\epsilon^{\prime}$ and there exists a diffeomorphism of $X_{r \leq \epsilon, r^{\prime} \geq \epsilon^{\prime}}$ onto $[0,1] \times X_{r=\epsilon}$ mapping $X_{r=\epsilon}$ onto $\{0\} \times X_{r=\epsilon}$ and $X_{r^{\prime}=\epsilon^{\prime}}$ onto $\{1\} \times X_{r=\epsilon}$.

Typically $r(z)$ is taken to be $|z|$, and then the link is just $X$ intersected with the round sphere $S_{\epsilon}^{2 n-1}$ centered at the origin, but sometimes it is convenient to work with a "stretched" link by choosing a different $r$.

For $\delta>0$, let $N_{\delta}$ denote the open $\delta$ neighborhood of the origin in $\mathbb{C}^{n-2}$. There exists a $\delta>0$ sufficiently small so that $f$ is a local submersion along $f^{-1}\left(N_{\delta}\right)_{r=\epsilon}$. Let $C_{f}$ denote the points of $f^{-1}\left(N_{\delta}\right)_{r<\epsilon}$ where $f$ fails to be a submersion. Let $D_{f}$ denote the image $f\left(C_{f}\right)$ in $N_{\delta}$. Then we have

Theorem $2.3([7])$. For $\delta>0$ as above,

(1) $f: f^{-1}\left(N_{\delta}\right)_{r=\epsilon} \rightarrow N_{\delta}$ is a $C^{\infty}$-trivial fiber bundle.

(2) $C_{f}$ has dimension $n-3$.

(3) $D_{f}$ is a hypersurface of dimension $n-3$ in $N_{\delta}$.

(4) The pair

$$
f:\left(f^{-1}\left(N_{\delta}-D_{f}\right)_{r \leq \epsilon}, f^{-1}\left(N_{\delta}-D_{f}\right)_{r=\epsilon}\right) \rightarrow N_{\delta}-D_{f}
$$

is a $C^{\infty}$-fiber bundle pair, each fiber of which is a complex analytic surface with boundary.

A smooth fiber $f^{-1}(\lambda)_{r \leq \epsilon}$ with $\lambda$ in $N_{\delta}-D_{f}$ is called a Milnor fiber of the singularity. Part (1) of Theorem 2.3 implies that the boundary of a Milnor fiber is diffeomorphic to the link. 
2.2. Splice diagrams. A splice diagram is a finite tree with no valence two vertices. Valence one vertices are called leaves, and vertices of valence greater than two are called nodes. The tree has integer edge weights on each edge at each node. An example of a typical splice diagram is

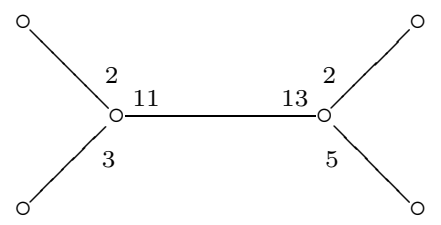

Splice diagrams classify integral homology sphere links:

Theorem 2.4 (2]). Integral homology sphere singularity links are in one-to-one correspondence with splice diagrams that satisfy

(1) weights around a node are positive and pairwise coprime;

(2) weights on an edge ending in a leaf are $>1$;

(3) all edge determinants are positive.

The third condition is a condition on the weights on any edge connecting two nodes. For such an edge $e$, the edge determinant, $\operatorname{det}(e)$, is the product of the weights on the edge, minus the product of the weights adjacent to the edge. For example, in the diagram

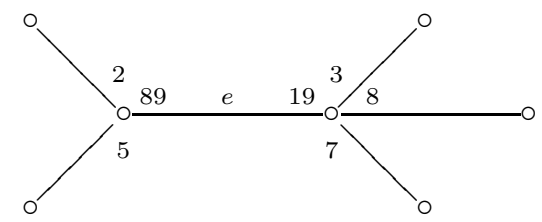

the edge determinant $\operatorname{det}(e)=89 \cdot 19-2 \cdot 5 \cdot 7 \cdot 8 \cdot 3=11$. The condition is that for every edge $e$ connecting two nodes, $\operatorname{det}(e)>0$.

To give the topological meaning of a splice diagram we first describe the manifold associated with a one node splice diagram and then describe an operation on two such manifolds called splicing that corresponds to joining two diagrams. A one node splice diagram with pairwise coprime edge weights $\alpha_{1}, \ldots, \alpha_{r}$ corresponds to the Brieskorn sphere, $\Sigma\left(\alpha_{1}, \ldots, \alpha_{r}\right)$. The Brieskorn sphere $\Sigma\left(\alpha_{1}, \ldots, \alpha_{r}\right)$ is the link of the Brieskorn complete intersection,

$$
V\left(\alpha_{1}, \ldots, \alpha_{r}\right)=\left\{z \in \mathbb{C}^{r} \mid a_{i 1} z_{1}^{\alpha_{1}}+\cdots+a_{i r} z_{r}^{\alpha_{r}}=0\right\}_{i=1}^{r-2},
$$

where the coefficient matrix $\left(a_{i j}\right)$ satisfies the condition that every $(r-2) \times(r-2)$ submatrix is non-singular [5]. For example, the Poincaré homology sphere $\Sigma(2,3,5)$ is the link of the singularity given by $x^{2}+y^{3}+z^{5}=0$ and is represented by the splice diagram

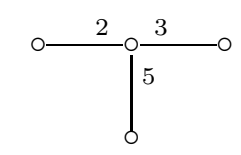

Each leaf $w_{i}$ of the diagram corresponds to the knot in $\Sigma\left(\alpha_{1}, \ldots, \alpha_{r}\right)$ cut out by setting the corresponding variable, $z_{i}$, equal to zero in $V\left(\alpha_{1}, \ldots, \alpha_{r}\right)$. We indicate 
this knot by placing an arrow at the leaf. For example,

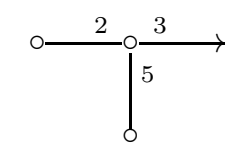

indicates the knot obtained by setting $y=0$ inside the link $\Sigma(2,3,5)$ of the singularity given by $x^{2}+y^{3}+z^{5}=0$.

A second description of the Brieskorn spheres is more familiar to 3-manifold topologists. The Brieskorn sphere $\Sigma\left(\alpha_{1}, \ldots, \alpha_{r}\right)$ is the genus zero Seifert fibered manifold $M\left(0 ;\left(\alpha_{1}, \beta_{1}\right),\left(\alpha_{2}, \beta_{2}\right), \ldots,\left(\alpha_{r}, \beta_{r}\right)\right)$, where $\beta_{1}, \ldots, \beta_{r}$ satisfy

$$
\alpha_{1} \cdots \alpha_{r} \cdot \sum_{i=1}^{r} \frac{\beta_{i}}{\alpha_{i}}=1
$$

(see [15]). This manifold can be constructed as follows. Start with the surface obtained by removing $r$ disjoint disks from a two sphere, $S=S^{2}-\left(D_{1}^{2} \cup \cdots \cup D_{r}^{2}\right)$, and take the Cartesian product with a circle, $S^{\prime}=S \times S^{1}$. Then the boundary of $S^{\prime}$ is $r$ disjoint tori $T_{1}, \ldots, T_{r}$, where $T_{i}=\partial D_{i}^{2} \times S^{1}$. The fundamental group of $S^{\prime}$ is generated by the boundaries of the $i$ removed disks in $S$, and one generator for the $S^{1}$ in the product $S \times S^{1}$. Glue a solid torus $H_{i}$ to each boundary component $T_{i}$ by the map $\phi_{i}: \partial H_{i} \rightarrow T_{i}$ that sends the meridian of $\partial H_{i}$ to $\alpha_{i} \cdot \partial D_{i}^{2}+\beta_{i} \cdot S^{1}$, where $\partial D_{i}^{2}$ and $S^{1}$ are the generators of $\pi_{1}\left(S^{\prime}\right)$. Then $M\left(0 ;\left(\alpha_{1}, \beta_{1}\right), \ldots,\left(\alpha_{r}, \beta_{r}\right)\right)=$ $S^{\prime} \cup_{\phi_{1}, \ldots \phi_{r}}\left(H_{1} \cup \cdots \cup H_{r}\right)$. The knot obtained by setting $z_{i}=0$ in the Brieskorn equations is the core of the $i$-th solid torus $H_{i}$ and is called the degree $\alpha_{i}$ singular fiber.

A diagram with more than one node can be obtained by joining together, or splicing, one node diagrams at their leaves. Topologically, this corresponds to removing a tubular neighborhood of each of the knots corresponding to the leaves along which the splicing occurs, and then gluing together the remaining knot exteriors along their torus boundaries matching meridian to longitude and longitude to meridian. For example

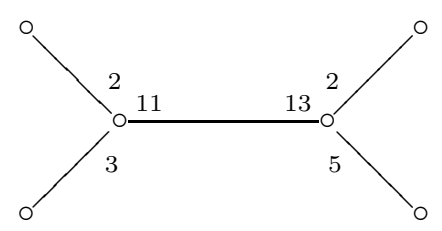

represents the Brieskorn spheres $\Sigma(2,3,11)$ and $\Sigma(2,5,13)$ spliced together along the degree 11 singular fiber of $\Sigma(2,3,11)$ and along the degree 13 singular fiber of $\Sigma(2,5,13)$. Such a diagram corresponds to an integral homology sphere link if and only if conditions (1) and (3) of Theorem 2.4 are satisfied. The decomposition of a homology sphere link into Brieskorn spheres is the well known JSJ-decomposition from 3-manifold topology.

The second condition of Theorem 2.4 is only necessary in order to guarantee a one-to-one correspondence between diagrams and homology sphere links, and it will often be useful to drop this condition. A diagram not satisfying this condition is called non-minimal. Call two splice diagrams that represent the same (up to orientation preserving homeomorphism) homology sphere equivalent diagrams. Any 
non-minimal splice diagram can be reduced to the unique equivalent minimal splice diagram by repeatedly applying the following operations [2]:

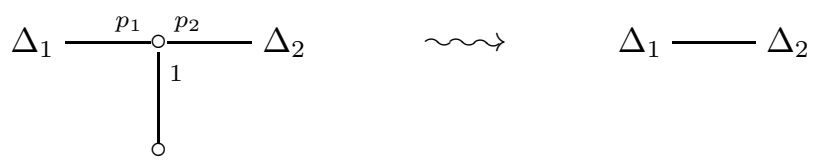

and, for $r>2$,

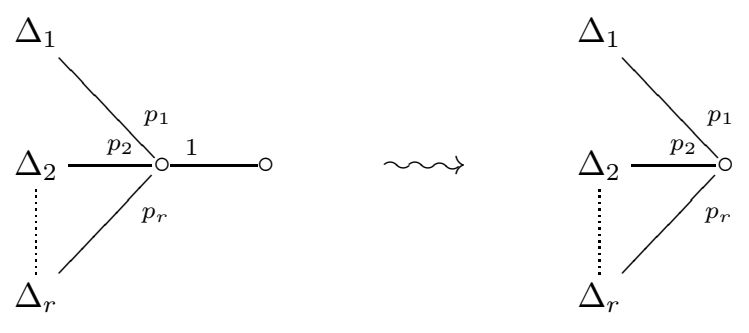

2.3. Splice type singularities and the Splice Type Conjecture. In [19] Neumann and Wahl give an algorithm to write explicit equations generalizing the Brieskorn equations for an isolated complete intersection surface singularity of given topology when the splice diagram for the link satisfies the additional semigroup condition. A singularity that can be obtained by this construction is said to be of splice type, and they make the Splice Type Conjecture (STC): Every complete intersection surface singularity with integral homology sphere link is of splice type 1

Equivalently, the minimal splice diagram of the link of an isolated complete intersection with homology sphere link must satisfy the semigroup condition. The conjecture has been confirmed for several families of singularities [14, 17, 18, 20,

Before describing the semigroup condition and defining the splice type equations, we recall some notation from [18, 19]. Let $\Delta$ be a splice diagram for a homology sphere $\Sigma$. Let $\delta_{v}$ denote the valence of a vertex $v$. For an edge $e$ at $v$, let $d_{v e}$ denote the weight on $e$ at $v$ and let $d_{v}$ denote the product $\Pi d_{v e}$ over all edges $e$ at $v$. For any two vertices, $v$ and $w$, let $l_{v w}$ be the product of weights adjacent to but not on the shortest path from $v$ to $w$. If $v$ and $w$ are leaves of the diagram, then they correspond to knots in $\Sigma$, and $l_{v w}$ is their linking number in $\Sigma$ [2]. Let $l_{v w}^{\prime}$ denote the same product, excluding weights adjacent to $v$ and $w$. Let $\Delta_{v e}$ be the subdiagram of $\Delta$ cut off from $v$ by $e$. For example, in the splice diagram,

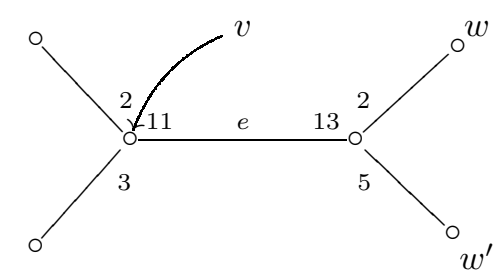

\footnotetext{
${ }^{1}$ Splice diagrams and splice type equations are also defined in the context of rational homology sphere links, where the splice type singularity associated with a splice diagram is the universal abelian cover of a singularity with the given topology [19]. The original conjecture was: Any $\mathbb{Q}$-Gorenstein singularity with $\mathbb{Q}$-homology sphere link has as universal abelian cover a splice type complete intersection. The conjecture has since proven false in this generality [8] 18, 19].
} 
TABLE 1. Splice diagram notation.

\begin{tabular}{|c|l|}
\hline$\delta_{v}$ & valence of $v$ \\
\hline$d_{v e}$ & weight on $e$ at $v$ \\
\hline$d_{v}$ & product of $d_{v e}$ over all edges $e$ at $v$ \\
\hline & $\begin{array}{l}\text { product of all weights adjacent to but not on the shortest } \\
\text { path from } v \text { to } w\end{array}$ \\
\hline$l_{v w}$ & $\begin{array}{l}\text { product of all weights adjacent to but not on the shortest } \\
\text { path from } v \text { to } w \text { excluding weights adjacent to } v \text { and } w\end{array}$ \\
\hline$l_{v w}^{\prime}$ & subdiagram of $\Delta$ cut off from $v$ by $e$ \\
\hline
\end{tabular}

$d_{v e}=11, d_{v}=66, l_{v w}=30, l_{v w}^{\prime}=5$ and

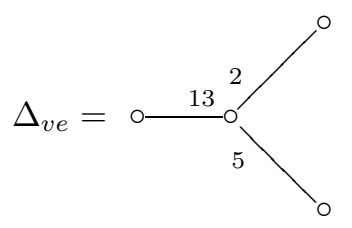

For reference these terms are collected in Table 1 .

A splice diagram $\Delta$ satisfies the semigroup condition if for each node $v$ and edge $e$ at $v$,

$$
\left.d_{v e} \in \mathbb{N}\left\langle l_{v w}^{\prime}\right| w \text { is a leaf of } \Delta_{v e}\right\rangle,
$$

or equivalently,

$$
\left.d_{v} \in \mathbb{N}\left\langle l_{v w}\right| w \text { is a leaf of } \Delta_{v e}\right\rangle .
$$

For example, in the diagram above the semigroup condition (2.1) at the node $v$ for the edge $e$ is $d_{v e}=11 \in \mathbb{N}\langle 5,2\rangle$. This condition is satisfied since $11=1 \cdot 5+3 \cdot 2$. The condition (2.1) is trivially satisfied for any edge leading to a leaf, since the relevant semigroup is then $\mathbb{N}$. The condition (2.2) at $v$ for the edge $e$ is $d_{v}=66 \in \mathbb{N}\langle 5,2\rangle$. This is satisfied since $66=6 \cdot 5+18 \cdot 2$. The condition (2.2) is also trivially satisfied for any edge leading to a leaf.

Now, suppose that $\Delta$ is a splice diagram satisfying the semigroup condition. Assign a variable $z_{w}$ to each leaf $w$ of $\Delta$. Neumann and Wahl give equations in these variables for a complete intersection surface with an isolated singularity at the origin such that the link of the singularity is the homology sphere specified by the diagram $\Delta$.

The system of equations consists of $\delta_{v}-2$ equations for each node $v$ of the diagram, giving a total of $n-2$ equations, where $n$ is the number of leaves of $\Delta$. Since $\Delta$ satisfies the semigroup condition, for each node $v$ and edge $e$ at $v$ we have

$$
d_{v e}=\sum \alpha_{v w} l_{v w}^{\prime}
$$


where the sum is taken over all leaves $w$ in $\Delta_{v e}$ (one could also use the equivalent form of the semigroup condition (2.2) $)$. Define an admissible monomial to be a monomial of the from

$$
\prod_{\text {leaves } w \text { of } \Delta_{v e}} z_{w}^{\alpha_{v w}} .
$$

Note that there may be several admissible monomials associated to a given node and edge, since there may be several choices of coefficients $\alpha_{v w}$ satisfying (2.3). For each edge $e$ at $v$, choose an admissible monomial $M_{v e}$ and form $\delta_{v}-2$ equations by

$$
\sum_{\text {edges } e \text { at } v} a_{i e} M_{v e}, \quad i=1, \ldots, \delta_{v}-2,
$$

where the $\delta_{v-2} \times \delta_{v}$ coefficient matrix $\left(a_{i e}\right)$ satisfies the condition that every $\delta_{v}-$ $2 \times \delta_{v}-2$ submatrix is non-singular. Repeating for each node $v$ of the diagram, we obtain a total of $n-2$ equations in $n$ variables. This system of equations is said to be of strict splice type.

For example, assigning variables $z_{1}, z_{2}, z_{3}, z_{4}$ to the leaves in the diagram

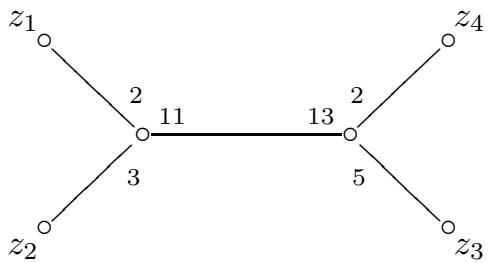

the admissible monomials at the left node are $z_{1}^{2}, z_{2}^{3}$ and $z_{3}^{3} z_{4}$. At the right node they are $z_{3}^{5}, z_{4}^{2}$ and $z_{1}^{3} z_{2}^{2}$ or $z_{1} z_{2}^{5}$. Thus, one choice of strict splice type equations for this diagram is

$$
\begin{aligned}
& z_{1}^{2}+z_{2}^{3}+z_{3}^{3} z_{4}=0 \\
& z_{3}^{5}+z_{4}^{2}+z_{1}^{3} z_{2}^{2}=0 .
\end{aligned}
$$

For another example see section 3.3 .

Notice that the equations at a node $v$ are weighted homogenous equations where the weight on the variable $z_{w}$ is $l_{v w}$ and the weight of each admissible monomial is $d_{v}$. In 14 it is shown that the only weighted homogenous normal surface singularities with integral homology sphere link are the Brieskorn complete intersections. Thus, as one can easily confirm by computation, in the one node case the strict splice type equations are the Brieskorn equations. One can view the semigroup condition and the resulting equations as motivated by the goal of constructing weighted homogenous equations at the nodes where the total weight matches the weight of the Brieskorn equations.

The weight $l_{v w}$ is called the $v$-weight of $z_{w}$. If one adds terms of $v$-weight strictly greater than $d_{v}$ to equation (2.4) to obtain

$$
\sum_{\text {edges } e \text { at } v} a_{i e} M_{v e}+H_{v i}, \quad i=1, \ldots, \delta_{v}-2,
$$

or makes a different choice of admissible monomials, then the resulting system defines a singularity that is an equisingular deformation of the one defined by the strict splice type equations (2.4). Here each $H_{v i}$ is a convergent power series in the $z_{w}$ consisting of monomial terms each of which has $v$-weight strictly greater than 
$d_{v}$. Such a system of equations is said to be of splice type, and Neumann and Wahl prove

Theorem 2.5 ([18, 19]). A system of equations of splice type defines an isolated complete intersection surface singularity with link $\Sigma$ the homology sphere specified by the splice diagram $\Delta$. Furthermore, the knot $\left(\Sigma, K_{w}\right)$ indicated by a leaf $w$ of $\Delta$ is cut out by setting the corresponding variable $z_{w}$ equal to zero, and this knot is fibered in $\Sigma$ with fibration given by $z_{w} /\left|z_{w}\right|: \Sigma-K_{w} \rightarrow S^{1}$.

Neumann and Wahl describe the construction of the splice type equations as "splicing the defining equations" [18, corresponding to splicing the links on the boundary. In section 2.4 we will see that in the case of a splice type singularity they conjecture a similar splicing of the Milnor fibers.

First, we recall some terminology and facts about the semigroups arising in the semigroup condition. These semigroups are always numeric semigroups. A numeric semigroup $\Gamma$ is a subsemigroup of $\mathbb{N}=\mathbb{Z}_{\geq 0}$ such that $\mathbb{N}-\Gamma$ is finite. The conductor of a numeric semigroup $\Gamma$ is defined to be the smallest number $c(\Gamma) \in \mathbb{N}$ such that $\gamma \geq c(\Gamma)$ implies $\gamma \in \Gamma$. For example, for $p, q \in \mathbb{N}$ coprime,

$$
c(\mathbb{N}\langle p, q\rangle)=(p-1)(q-1) .
$$

We will also need the following result on the conductor of certain semigroups.

Lemma 2.6 ([18]). Suppose $\Gamma_{i}$ are numeric semigroups for $i=1, \ldots, n$ and $p_{1}, \ldots, p_{n}$ are pairwise coprime integers with $p_{i} \in \Gamma_{i}$. Write $P=p_{1} \cdots p_{n}$ and $P_{i}=P / p_{i}$. Let

$$
\Gamma=P_{1} \Gamma_{1}+\cdots+P_{n} \Gamma_{n}
$$

Then

$$
c(\Gamma)=\sum_{i=1}^{n} P_{i}\left(c\left(\Gamma_{i}\right)-1\right)+(n-1) P+1 .
$$

2.4. The Milnor Fiber Conjecture. The MFC describes a topological construction of the Milnor fiber for splice type singularities by extending the operation of splicing homology sphere links to a "splicing" of the associated Milnor fibers. The Casson invariant is additive under splicing, as is the signature of the Milnor fiber under the conjectured topological construction. The CIC is known for Brieskorn complete intersections, and thus the MFC implies the CIC for singularities of splice type [18. However, the MFC provides more than the CIC: It gives a complete picture of the topology of $F$ in terms of the JSJ-decomposition of the link.

We now describe the conjectured construction (see Figure 1). Let $\Sigma$ be the link of a splice type singularity specified by a diagram $\Delta$ that satisfies the semigroup condition. Decompose $\Sigma$ as the splice of two homology spheres $\Sigma_{1}$ and $\Sigma_{2}$ corresponding to a decomposition of the diagram into $\Delta_{1}$ and $\Delta_{2}$. Both $\Delta_{1}$ and $\Delta_{2}$ also satisfy the semigroup condition, and thus $\Sigma_{1}$ and $\Sigma_{2}$ are the links of splice type singularities with Milnor fibers $F_{1}$ and $F_{2}$ where $\partial F_{i}=\Sigma_{i}$. Let $w_{i} \in \Delta_{i}$ be the leaves along which the splicing occurs. Then $w_{i}$ corresponds to a knot $K_{i} \subset \Sigma_{i}$ given by setting the corresponding variable to zero in the splice type equations.

The knot $K_{i}$ is fibered in $\Sigma_{i}$. Let $G_{i}$ denote the fiber of $K_{i} \subset \Sigma_{i}$, so $G_{i}$ is a real 2-manifold in $\Sigma_{i}$ with boundary $K_{i}$. Push $G_{i}$ into $F_{i}$, keeping the boundary $\partial G_{i}=K_{i} \subset \Sigma_{i}$ fixed, so that it is properly embedded. Let $F_{i}^{\circ}$ denote the result of removing an open tubular neighborhood, $G_{i} \times \stackrel{\circ}{D^{2}}$, of this properly embedded 
$G_{i}$. Then the boundary of $F_{i}^{\circ}$ consists of the exterior of the knot $K_{i}, \Sigma_{i}-N\left(K_{i}\right)$, and $G_{i} \times S^{1}$. Join $F_{1}^{\circ}$ and $F_{2}^{\circ}$ by gluing in a $G_{1} \times G_{2}$ as follows. The boundary of $G_{1} \times G_{2}$ is $\left(\partial G_{1} \times G_{2}\right) \cup\left(G_{1} \times \partial G_{2}\right)=\left(K_{1} \times G_{2}\right) \cup\left(G_{1} \times K_{2}\right)=S^{1} \times G_{2} \cup G_{1} \times S^{1}$. Glue the $G_{1} \times S^{1}$ to the corresponding piece of the boundary of $F_{1}^{\circ}$ and similarly glue the $S^{1} \times G_{2}$ to the corresponding piece of the boundary of $F_{2}^{\circ}$. Identify the rest of the boundary of $F_{1}^{\circ}$, the exterior of $K_{1} \subset \Sigma_{1}$, with the exterior of the $K_{2} \subset \Sigma_{2}$ portion of the boundary of $F_{2}^{\circ}$ by matching meridian to longitude and longitude to meridian as in the splicing construction. Then $\bar{F}$ has boundary $\Sigma$ and the Milnor Fiber Conjecture is:

Conjecture 2.7 ([18]). The Milnor fiber $F$ of the splice type singularity specified by $\Delta$ is homeomorphic to $\bar{F}$.

Note that this conjecture applies to a diagram $\Delta$ satisfying the semigroup condition with a distinguished edge $e$ of $\Delta$. We will say that the MFC holds for $\Delta$ if the construction of $\bar{F}$ described above corresponding to decomposing $\Delta$ along any of its edges yields $F$. We show in section 3.2 that if the MFC holds for a diagram $\Delta$, then it holds for any equivalent diagram that also satisfies the semigroup condition.

\section{ITERATED BRANCHED CYCLIC COVERS And the Milnor Fiber Conjecture}

In 18 Neumann and Wahl proved the MFC for hypersurface singularities given by an equation of the form $f(x, y)+z^{n}=0$. The link of such a singularity is a branched cyclic cover of $S^{3}$ branched along the graph knot cut out by the plane curve $f(x, y)=0$. In section 3.1 we generalize this result to iterated branched cyclic covers of homology sphere links branched along graph knots (Theorem 3.4).

In order to widen the class of examples to which this theorem applies, in section 3.2 we prove that if the MFC holds for a given diagram, then the MFC also holds for any equivalent diagram that also satisfies the semigroup condition (Theorem 3.8).

Beginning with a hypersurface $f(x, y)+z^{n}=0$, and repeatedly applying Theorems 3.4 and 3.8, one has an abundance of singularities for which the MFC holds. These are the only known examples. In section 3.3 we go through such an example in detail. In section 3.4 we discuss the relationship between these examples and the Splice Type Conjecture.

3.1. Branched cyclic covers and the Milnor fiber. Neumann and Wahl give a necessary and sufficient condition for the $k$-fold branched cyclic cover of a homology sphere link $\Sigma^{\prime}$ branched along a graph knot $K$ to be a homology sphere link $\Sigma$ in [16. We recall that condition here.

Suppose $\Sigma^{\prime}$ is a homology sphere given by a splice diagram $\Delta$, and $\left(\Sigma^{\prime}, K\right)$ is a graph knot indicated by a distinguished leaf $*$ of $\Delta$. Each edge of $\Delta$ has either one or two edge weights attached to it, depending on if the edge connects a node and a leaf of the diagram or connects two nodes. Following Neumann and Wahl, call the weight on an edge "near" or "far" if it is on the end of the edge closest to or furthest from $*$ in $\Delta$. For example, in the following diagram the weights $n$ are near 


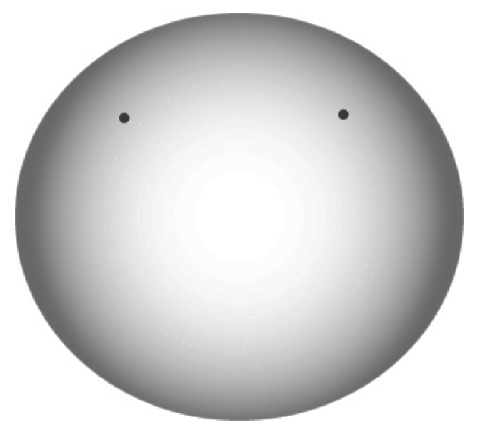

a. $\left(\Sigma_{i}, K_{i}\right)$

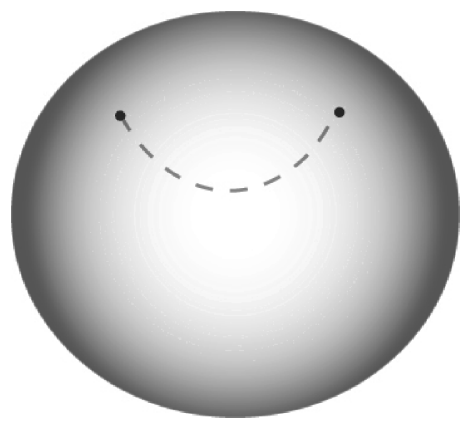

c. $G_{i}$ pushed into $F_{i}$

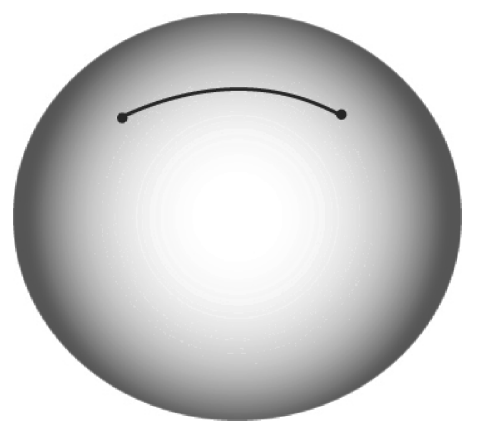

b. $\left(\Sigma_{i}, K_{i}\right)$ and $G_{i}$

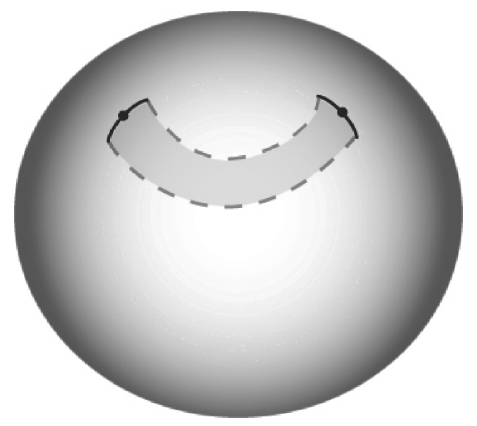

d. $F_{i}^{\circ}$

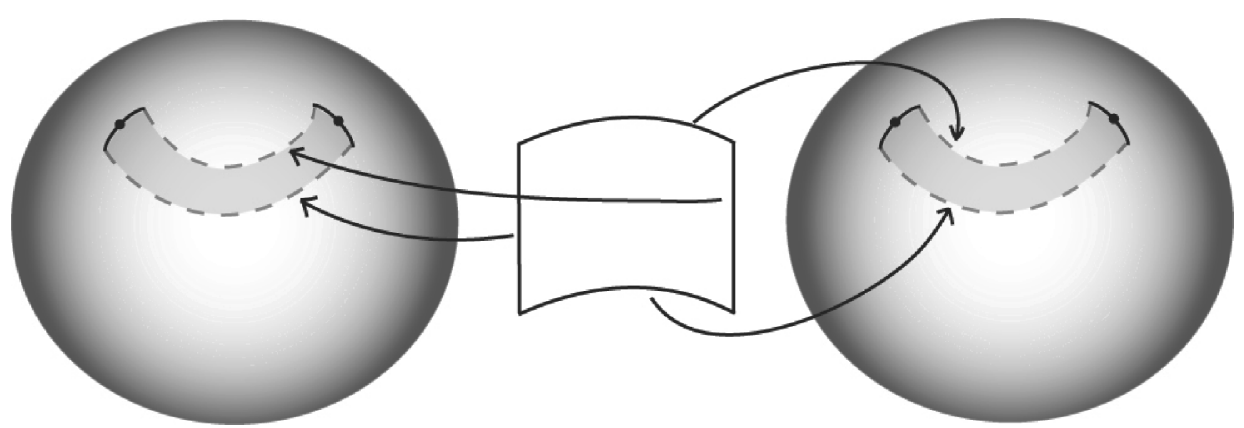

e. Glue $G_{1} \times G_{2}$ to $F_{1}^{\circ}$ and $F_{2}^{\circ}$ along the boundary.

FiguRE 1. The steps in the MFC construction.

with respect to the leaf $*$ and the weights $f$ are far:

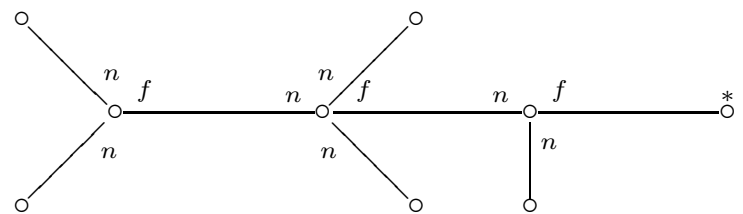

Denote by $\Delta(*, k)$ the diagram obtained from $\Delta$ by multiplying all far edge weights with respect to $*$ by $k$. 
Theorem 3.1 ([16]). The $k$-fold branched cyclic cover of $\Sigma^{\prime}$ branched along $K$ is a homology sphere $\Sigma$ if and only if $k$ is prime to all near weights in $\Delta$, and $\Sigma$ is then given by the splice diagram $\Delta(*, k)$.

For example, we saw in section 2.3 that the homology sphere $\Sigma^{\prime}$ specified by the diagram

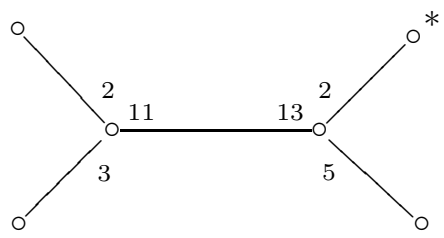

is the link of the splice type singularity given by

$$
\begin{aligned}
& z_{1}^{2}+z_{2}^{3}+z_{3}^{3} z_{4}=0 \\
& z_{3}^{5}+z_{4}^{2}+z_{1}^{3} z_{2}^{2}=0 .
\end{aligned}
$$

The knot $K$ indicated by the leaf $*$ is cut out by $z_{4}=0$. The edge weights near with respect to $*$ are $2,3,5$ and 13 , so the 7 -fold branched cyclic cover of $\Sigma^{\prime}$ with branch set $K$ is the homology sphere link $\Sigma$ specified by the diagram

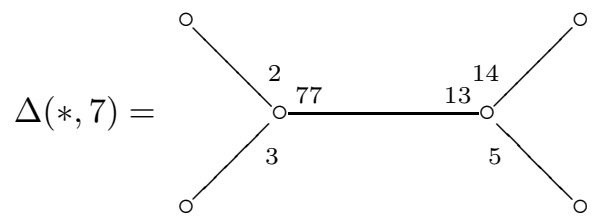

The splice type equations for this diagram can be chosen as

$$
\begin{array}{r}
z_{1}^{2}+z_{2}^{3}+z_{3}^{3} z_{4}^{7}=0, \\
z_{3}^{5}+z_{4}^{14}+z_{1}^{3} z_{2}^{2}=0,
\end{array}
$$

and in these coordinates the branched cyclic covering $\phi: \Sigma \rightarrow \Sigma^{\prime}$ is just $\left(z_{1}, z_{2}, z_{3}, z_{4}\right)$ $\mapsto\left(z_{1}, z_{2}, z_{3}, z_{4}^{7}\right)$.

Now, suppose that $\Delta$ satisfies the semigroup condition and that the MFC holds for $\Delta$. We will show that the MFC holds for $\Delta(*, k)$ when $\Delta(*, k)$ corresponds to a homology sphere link as in Theorem 3.1. First, we prove that the branched cyclic covering of the links $\Sigma \rightarrow \Sigma^{\prime}$ extends to a branched cyclic covering of the corresponding Milnor fibers $F \rightarrow F^{\prime}$.

Since $\Sigma^{\prime}$ is the link of a splice type singularity and $K$ corresponds to the leaf $*$ of $\Delta, K$ is cut out in $\Sigma^{\prime}$ by setting the variable $z_{*}$ corresponding to $*$ in some splice type equations for $\Delta$ equal to zero. This knot is fibered with fibration given by $z_{*} /\left|z_{*}\right|: \Sigma^{\prime}-K \rightarrow S^{1}$ (Theorem 2.5). Let $G$ denote a fiber of this fibration. Then,

Theorem 3.2. $F$ is a $k$-fold branched cyclic cover of $F^{\prime}$ branched along a copy of $G$ pushed into $F^{\prime}$ so that it is properly embedded.

This theorem is a generalization of the hypersurface case proved in 6. Before proving Theorem 3.2, we prove the following lemma, which describes how the splice type equations for $\Delta(*, k)$ can be obtained from equations for $\Delta$.

Lemma 3.3. Replacing $z_{*}$ by $z_{*}^{k}$ in the splice type equations for $\Delta$ gives splice type equations for $\Delta(*, k)$. 
Proof. Since both $\Delta$ and $\Delta(*, k)$ have the same underlying graph and differ only in their weights, we will refer to nodes, edges and leaves in both of them simultaneously. For any node $v$ and edge $e$ at $v$ let $d_{v e}[\Delta]$ denote the weight on $e$ at $v$ in $\Delta$ and $d_{v e}[\Delta(*, k)]$ the corresponding weight in $\Delta(*, k)$. Then for any node $v$ and edge $e$ at $v$,

$$
d_{v e}[\Delta(*, k)]= \begin{cases}d_{v e}[\Delta], & * \notin \Delta_{v e}, \\ k d_{v e}[\Delta], & * \in \Delta_{v e}\end{cases}
$$

For a node $v$ and leaf $w$, let $l_{v w}^{\prime}[\Delta]$ denote the product of all the weights in $\Delta$ adjacent to but not on the shortest path from $v$ to $w$, not including the weights around $v$ and $w$ and let $l_{v w}^{\prime}[\Delta(*, k)]$ denote the same product using the weights from $\Delta(*, k)$. Then, for any node $v$ and edge $e$ at $v$, for all leaves $w \in \Delta_{v e}$,

$$
l_{v w}^{\prime}[\Delta(*, k)]= \begin{cases}l_{v w}^{\prime}[\Delta], & * \notin \Delta_{v e} \text { or } w=*, \\ k l_{v w}^{\prime}[\Delta], & * \in \Delta_{v e} \text { and } w \neq * .\end{cases}
$$

Now, fix a node $v$. Let $\delta_{v}$ be the number of edges at $v$. The semigroup condition on the weights of $\Delta$ allows us to write

$$
d_{v e}[\Delta]=\sum_{w \in \Delta_{v e}} \alpha_{v w} l_{v w}^{\prime}[\Delta] .
$$

Recall that an admissible monomial associated to the edge $e$ at $v$ is a monomial of the form

$$
\prod_{\text {leaves } w \text { of } \Delta_{v e}} z_{w}^{\alpha_{v w}} .
$$

For each edge $e$ at $v$, choose an admissible monomial for $\Delta, M_{v e}[\Delta]$, and consider the $\delta_{v}-2$ equations

$$
\sum_{e} a_{i e} M_{v e}[\Delta]=0, \quad i=1, \ldots, \delta_{v}-2,
$$

for sufficiently general coefficients $a_{i e}$. Repeating this for each node $v \in \Delta$ we obtain splice type equations for $\Delta$.

Now, if $* \notin \Delta_{v e}$, then

$$
\begin{aligned}
d_{v e}[\Delta(*, k)]=d_{v e}[\Delta] & =\sum_{w \in \Delta_{v e}} \alpha_{v w} l_{v w}^{\prime} \\
& =\sum_{w \in \Delta_{v e}} \alpha_{v w} l_{v w}^{\prime}[\Delta(*, k)],
\end{aligned}
$$

and thus one can choose the admissible monomial $M_{v e}[\Delta(*, k)]$ for $\Delta(*, k)$ to be the same as $M_{v e}[\Delta]$. Since $* \notin \Delta_{v e}$, the variable $z_{*}$ does not occur in $M_{v e}[\Delta(*, k)]=$ $M_{v e}[\Delta]$.

If $* \in \Delta_{v e}$, then,

$$
\begin{aligned}
d_{v e}[\Delta(*, k)]=k d_{v e}[\Delta] & =k \sum_{w \in \Delta_{v e}} \alpha_{v w} l_{v w}^{\prime}[\Delta] \\
& =\sum_{w \in \Delta_{v e}} \alpha_{v w} k l_{v w}^{\prime}[\Delta] \\
& =\left(\sum_{w \neq * \in \Delta_{v e}} \alpha_{v w} l_{v w}^{\prime}[\Delta(*, k)]\right)+\alpha_{v *} k l_{v *}^{\prime}[\Delta(*, k)] .
\end{aligned}
$$




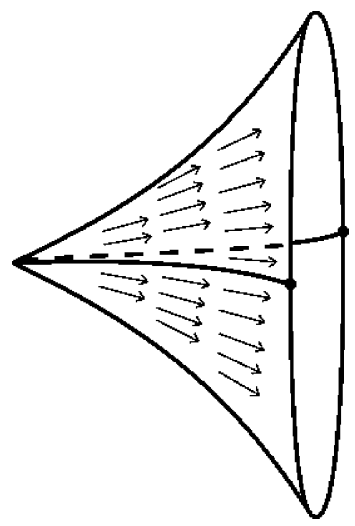

Figure 2. The vector field $v$ on $X_{r \leq \epsilon}^{\prime}-p_{n}^{-1}(0)$.

So, $\Delta(*, k)$ satisfies the semigroup condition, and the admissible monomial $M_{v e}[\Delta(*, k)]$ can be chosen to be the same as the admissible monomial $M_{v e}[\Delta]$, except that the power on $z_{*}$ is changed from $\alpha_{v *}$ to $\alpha_{v *} k$. Thus, the splice type equations for $\Delta(*, k)$ are obtained from those for $\Delta$ simply by replacing $z_{*}$ with $z_{*}^{k}$ everywhere it occurs.

We now prove Theorem 3.2 ,

Proof. Suppose that $f: \mathbb{C}^{n} \rightarrow \mathbb{C}^{n-2}$ are splice type equations corresponding to $\Delta$ and that $z_{*}=z_{n}$ in these equations. By Lemma 3.3, $f_{(k)}: \mathbb{C}^{n} \rightarrow \mathbb{C}^{n-2}$ given by $f_{(k)}\left(z_{1}, \ldots, z_{n}\right)=f\left(z_{1}, \ldots, z_{n-1}, z_{n}^{k}\right)$ are splice type equations for $\Delta(*, k)$. Let

$$
X=f_{(k)}^{-1}(0)
$$

and

$$
X^{\prime}=f^{-1}(0)
$$

be the corresponding splice type complete intersections.

Let $r: \mathbb{C}^{n} \rightarrow[0, \infty)$ be given by

$$
r(z)=\left|z_{1}\right|^{2}+\cdots+\left|z_{n-1}\right|^{2}+\left|z_{n}\right|^{2 k}
$$

and let $r^{\prime}: \mathbb{C}^{n} \rightarrow[0, \infty)$ be given by

$$
r^{\prime}(z)=|z|^{2} .
$$

Choose $\epsilon^{2}$ sufficiently small so that Proposition 2.1 applies to both $(X, o), r$ and $\left(X^{\prime}, o\right), r^{\prime}$, and so that $\left.r^{\prime}\right|_{\left\{z \in X^{\prime} \mid z_{n}=0\right\}}$ has no critical values in $\left(0, \epsilon^{2}\right]$.

Let $p_{n}: \mathbb{C}^{n} \rightarrow \mathbb{C}$ denote projection onto the $n$-th coordinate. In [4] Lemma 3.5, Hamm constructs a vector field $v$ on $X_{r^{\prime} \leq \epsilon^{2}}^{\prime}-p_{n}^{-1}(0)$ (see Figure 2) such that

(1) the real part of $\langle v(z), z\rangle$ is positive and

(2) $\left\langle v(z), \operatorname{grad} \log p_{n}(z)\right\rangle=1$.

The first condition ensures that $r^{\prime}(z)$ increases along integral curves of $v$ and the second guarantees that $\arg z_{n}=\frac{z_{n}}{\left|z_{n}\right|}$ remains constant and $\left|z_{n}\right|$ increases along integral curves of $v\left(\right.$ since grad $\left.\log p_{n}(z)=\frac{z_{n}}{\left|z_{n}\right|^{2}} \operatorname{grad} p_{n}(z)\right)$. 


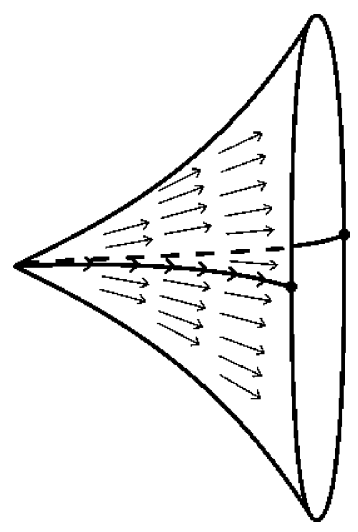

Figure 3. The vector field $\tilde{v}$ extends $v$ to $X_{r \leq \epsilon}^{\prime}-o$.

This vector field can be extended to a vector field $\tilde{v}$ on all of $X_{r^{\prime} \leq \epsilon^{2}}^{\prime}-o$ (see Figure 3) so that the first condition holds, the second condition holds when $z_{n} \neq 0$, and so that $\tilde{v}(z)$ is tangent to $p_{n}^{-1}(0)$ when $z_{n}=0$. The vector field can be constructed locally at points of $p_{n}^{-1}(0)$ and then patched together with $v$ using a partition of unity to obtain $\tilde{v}$ :

For $z \in X_{r^{\prime} \leq \epsilon^{2}}^{\prime}-o$ with $z_{n}=0$, the map

$$
\Phi=\left(f, p_{n}, r^{\prime}\right): \mathbb{C}^{n}=\mathbb{R}^{2 n} \rightarrow \mathbb{C}^{n-2} \times \mathbb{C} \times \mathbb{R}=\mathbb{R}^{2 n-1}
$$

is regular in a neighborhood $U$ of $z$. So, there exist real local coordinates $u_{1}, \ldots, u_{2 n}$ on $U$ so that $\Phi\left(u_{1}, \ldots, u_{n}\right)=\left(u_{1}, \ldots, u_{2 n-1}\right)$ in these coordinates. In these coordinates the vector field

$$
\sum_{i=1}^{2 n-2} u_{i} \frac{\partial}{\partial u_{i}}+\frac{\partial}{\partial u_{2 n-1}}
$$

satisfies the desired conditions. Patching this together with $v$, using a partition of unity, gives $\tilde{v}$.

Then for $\eta \in \mathbb{C}$ sufficiently close to the origin, pushing out along integral curves of $\tilde{v}$ provides an isotopy of $\left\{z \in X_{r^{\prime} \leq \epsilon^{2}}^{\prime} \mid z_{n}=\eta\right\}$ to a fiber $\frac{z_{n}}{\left|z_{n}\right|}=$ constant of $K$ in $\Sigma^{\prime}$ (see Figure 4 ).

Fix such an $\eta$. Since all the properties of $\tilde{v}$ are local, $\tilde{v}$ can be extended except near the origin to a vector field $w$ on a neighborhood of $X_{r^{\prime} \leq \epsilon^{2}}^{\prime}$ so that

(1) $w$ is tangent to the fibers of $f$,

(2) the real part of $\langle w(z), z\rangle$ is positive,

(3) $\left\langle w(z), \operatorname{grad} \log p_{n}(z)\right\rangle=1$ when $z_{n} \neq 0$ and

(4) $w(z)$ is tangent to $p_{n}^{-1}(0)$ when $z_{n}=0$.

Let $w$ be such an extension of $\tilde{v}$ on a neighborhood $W$ of $X_{r^{\prime} \leq \epsilon^{2}}^{\prime}-o$, outside of $\left\{\left.z \in \mathbb{C}^{n}|| z\right|^{2}<\epsilon_{0}^{2}\right\}$ for some $\epsilon_{0}^{2}<|\eta|^{2}$ (see Figure 5).

Choose $\delta>0$ so that Theorem 2.3 applies to $f_{(k)}, r, \epsilon^{2}$. Choose $\delta^{\prime}>0$ so that Theorem 2.3 applies to $f, r^{\prime}, \epsilon^{2}$. Then choose $\lambda \in N_{\delta} \cap N_{\delta^{\prime}}$ so that

$$
F=f_{(k)}^{-1}(\lambda)_{r \leq \epsilon^{2}}
$$




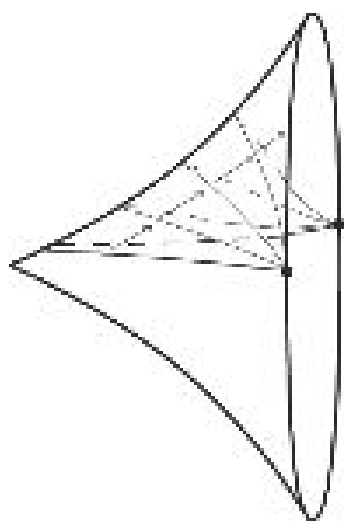

Figure 4. Integral curves of $\tilde{v}$ provide an isotopy of $\{z \in$ $\left.X_{r^{\prime} \leq \epsilon^{2}}^{\prime} \mid z_{n}=\eta\right\}$ to a fiber of $K$.

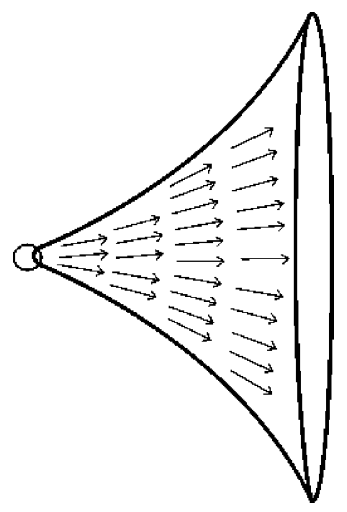

Figure 5. The vector field $\tilde{v}$ extends to a vector field $w$ on nearby fibers, away from the origin.

is a Milnor fiber for $(X, o)$ and

$$
F^{\prime}=f^{-1}(\lambda)_{r^{\prime} \leq \epsilon^{2}}
$$

is a Milnor fiber for $\left(X^{\prime}, o\right)$ and $F_{r^{\prime} \geq \epsilon_{0}^{2}}^{\prime} \subset W$ and so that $B_{\eta}=\left\{z \in F^{\prime} \mid z_{n}=\eta\right\}$ is smooth.

The map $p: F \rightarrow F^{\prime}$ given by $\left(z_{1}, \ldots, z_{n}\right) \mapsto\left(z_{1}, \ldots, z_{n-1}, z_{n}^{k}\right)$ is a $k$-fold branched cyclic covering with branch set $B_{0}:=\left\{z \in F^{\prime} \mid z_{n}=0\right\}$. It remains to show that $B_{0}$ is a copy of the fiber $G$ of $K$ in $\Sigma^{\prime}$ that has been pushed into $F^{\prime}$ so that it is properly embedded. Since the integral curves of $w$ provide an isotopy of $B_{\eta}$ to $G=\left\{z \in \Sigma^{\prime}=F_{r^{\prime}=\epsilon^{2}}^{\prime} \mid \frac{z_{n}}{\left|z_{n}\right|}=\frac{\eta}{|\eta|}\right\}$ in $F^{\prime}$ (see Figure 6), it is sufficient to show that $B_{0}$ is isotopic to $B_{\eta}$ in $F^{\prime}$.

Consider the set $A$ consisting of all points $\alpha \in \mathbb{C}$ for which $B_{\alpha}:=\left\{z \in F^{\prime} \mid z_{n}=\right.$ $\alpha\}$ is not smooth. This is a sub-variety of $\mathbb{C}$ not containing the origin and $\eta$, and is thus zero-dimensional. So, there is a path $\gamma:[0,1] \rightarrow \mathbb{C}$, diffeomorphic onto 


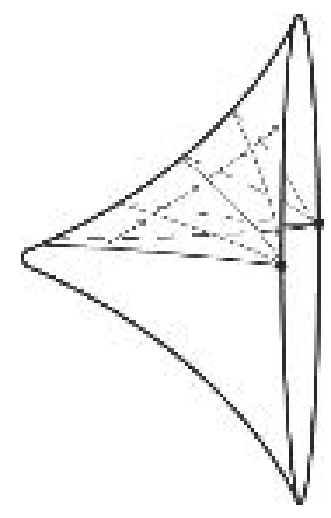

Figure 6. Integral curves of $w$ provide an isotopy of $B_{\eta}$ to $G$ in $F^{\prime}$.

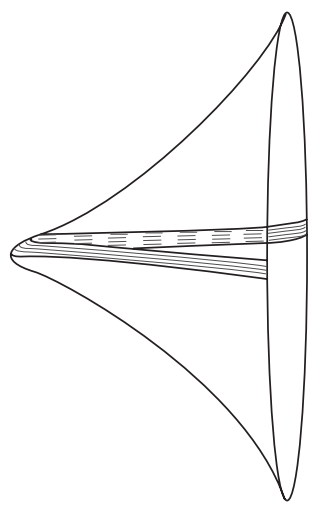

Figure 7. By Ehresmann's fibration theorem, $B_{0}$ is isotopic to $B_{\eta}$.

its image, such that $\gamma(0)=o, \gamma(1)=\delta$ and $\gamma(t) \notin A$ for any $t$. Let $B_{\gamma(t)}=$ $\left\{z \in F^{\prime} \mid z_{n}=\gamma(t)\right\}$ and $B=\bigcup_{t \in[0,1]} B_{\gamma(t)} \subset F^{\prime}$. Then $\phi: B \rightarrow[0,1]$ given by $z \mapsto \gamma^{-1}\left(z_{n}\right)$ is a proper submersion and thus, by Ehresmann's fibration theorem, is a $C^{\infty}$-trivial fibration and the fibers $\phi^{-1}(0)=B_{0}$ and $\phi^{-1}(1)=B_{\eta}$ are isotopic (see Figure 7).

Then, the main theorem is:

Theorem 3.4. Suppose that $\Delta$ is a splice diagram satisfying the semigroup condition and that the Milnor Fiber Conjecture holds for $\Delta$. Let $* \in \Delta$ be a distinguished leaf. If $k \in \mathbb{N}$ is pairwise coprime to all near edge weights of $\Delta$ with respect to $*$, then the Milnor Fiber Conjecture also holds for $\Delta(*, k)$.

Proof. Let $\left(X^{\prime}, o\right)$ be a splice type singularity corresponding to $\Delta$ and $(X, o)$ a splice type singularity corresponding to $\Delta(*, k)$. Let $\Sigma^{\prime}$ and $F^{\prime}$ be the link and Milnor fiber of $\left(X^{\prime}, o\right)$ and $\Sigma$ and $F$ be the link and Milnor fiber of $(X, o)$. By Theorem 2.5. $\Sigma^{\prime}$ is the homology sphere specified by $\Delta$ and $\Sigma$ is the homology sphere specified 
TABLE 2. Corresponding objects in the base and in the cover.

\begin{tabular}{|r|c|c|}
\hline singularity & $\left(X^{\prime}, o\right)$ & $(X, o)$ \\
\hline diagram & $\Delta=\Delta_{1}^{\prime} \stackrel{w_{1}}{\longleftrightarrow} \stackrel{w_{2}}{\longleftarrow} \Delta_{2}^{\prime}$ & $\Delta(*, k)=\Delta_{1} \stackrel{w_{1}}{\longleftrightarrow} \stackrel{w}{2}_{2} \Delta_{2}$ \\
\hline link & $\Sigma^{\prime}=\Sigma_{1}^{\prime} \stackrel{K_{1}^{\prime}}{\longleftrightarrow} \stackrel{K_{2}^{\prime}}{\longleftarrow} \Sigma_{2}^{\prime}$ & $\Sigma=\Sigma_{1} \stackrel{K_{1} K_{2}}{\longleftrightarrow} \Sigma_{2}$ \\
\hline Milnor fiber & $F^{\prime}=\left(F_{1}^{\prime}\right)^{\circ} \cup G_{1}^{\prime} \times G_{2}^{\prime} \cup\left(F_{2}^{\prime}\right)^{\circ}$ & $F \stackrel{?}{=} F_{1}^{\circ} \cup G_{1} \times G_{2} \cup F_{2}^{\circ}$ \\
\hline
\end{tabular}

by $\Delta(*, k)$. Let $\left(\Sigma^{\prime}, K\right)$ be the knot specified by $* \in \Delta$, so $\Sigma$ is the $k$-fold branched cyclic cover of $\Sigma^{\prime}$ branched along $K$.

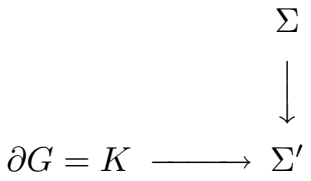

Let $G$ be the fiber of $K$ in $\Sigma^{\prime}$. By Theorem 3.2, $F$ is a $k$-fold branched cyclic cover of $F^{\prime}$ branched along a copy of $G$ pushed into $F^{\prime}$ so that it is properly embedded.

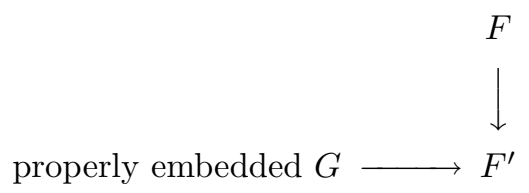

Decompose $\Sigma$ as the splice of two homology spheres $\Sigma_{1}$ and $\Sigma_{2}$ corresponding to a decomposition of the diagram $\Delta(*, k)$ as

$$
\Delta(*, k)=\Delta_{1} \stackrel{w_{1}}{\longrightarrow} \stackrel{w_{2}}{\longleftarrow} \Delta_{2} .
$$

Without loss of generality suppose that the leaf $*$ is in $\Delta_{2}$. Since $\Delta$ and $\Delta(*, k)$ have the same underlying graph, there is a corresponding decomposition

$$
\Delta=\Delta_{1}^{\prime} \stackrel{w_{1}}{\longrightarrow} \stackrel{w_{2}}{\longleftarrow} \Delta_{2}^{\prime}
$$

where $\Delta_{1}=\Delta_{1}^{\prime}\left(w_{1}, k\right)$ and $\Delta_{2}=\Delta_{2}^{\prime}(*, k)$. The diagrams $\Delta_{i}$ and $\Delta_{i}^{\prime}$, must also satisfy the semigroup condition, and thus $\Sigma_{i}$ and $\Sigma_{i}^{\prime}$ are links of splice type singularities, with Milnor fibers $F_{i}$ and $F_{i}^{\prime}$ respectively. The leaves $w_{i}$ of $\Delta_{i}$ and $\Delta_{i}^{\prime}$ correspond to fibered knots $\left(\Sigma_{i}, K_{i}\right)$ and $\left(\Sigma_{i}^{\prime}, K_{i}^{\prime}\right)$, along which the splicing occurs. Let $G_{i}$ be the fiber of the knot $\left(\Sigma_{i}, K_{i}\right)$ and let $G_{i}^{\prime}$ be the fiber of the knot $\left(\Sigma_{i}^{\prime}, K_{i}^{\prime}\right)$. By assumption the MFC holds for $\Delta$, and thus $F^{\prime}$ decomposes as

$$
F^{\prime}=\left(F_{1}^{\prime}\right)^{\circ} \cup G_{1}^{\prime} \times G_{2}^{\prime} \cup\left(F_{2}^{\prime}\right)^{\circ},
$$

where $\left(F_{i}^{\prime}\right)^{\circ}$ is the fiber $F_{i}^{\prime}$ minus a tubular neighborhood of a properly embedded fiber $G_{i}^{\prime}$ of $K_{i}^{\prime}$ as described in section 2.4.

We will show that on taking the branched cyclic cover of $F^{\prime}$ branched along a properly embedded $G$, the pieces in the MFC decomposition (3.1) for $F^{\prime}$ lift to the desired pieces in the conjectured decomposition of $F$ :

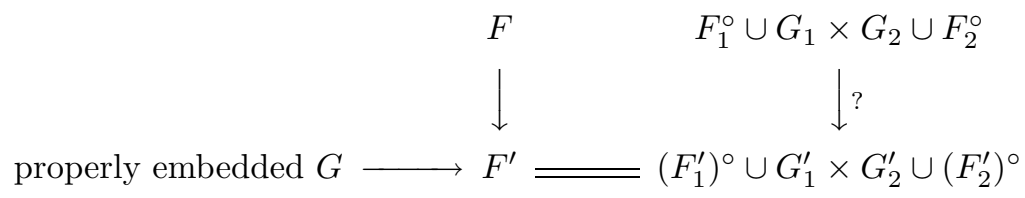

Table 2 summarizes the situation. 
First, we describe the topology of the branch set $G$ in terms of the splice decomposition of $\Sigma^{\prime}$ and then position it with respect to the decomposition (3.1). Let $l=l_{* w_{2}}$ be the linking number of the knot $K$ and $K_{2}^{\prime}$ in $\Sigma_{2}^{\prime}[2$. Then, as shown in [2], the splice decomposition $\Sigma^{\prime}=\Sigma_{1}^{\prime} \stackrel{K_{1}^{\prime}}{\longrightarrow} \stackrel{K_{2}^{\prime}}{\longleftarrow} \Sigma_{2}^{\prime}$ leads to a description of $G$ as $l$ parallel copies of $G_{1}^{\prime}$ and one copy of the fiber corresponding to the diagram $\Delta_{2}^{\prime} \longrightarrow^{*}$ punctured $l$ times.

To see this, note that from the definition of linking number the knot $K_{2}^{\prime}$ in $\Sigma_{2}^{\prime}$ intersects each fiber of $\Delta_{2}^{\prime} \longrightarrow{ }^{*} l$ times, so when we remove a tubular neighborhood of $K_{2}^{\prime}$ in $\Sigma_{2}^{\prime}$ each fiber of $\Delta_{2}^{\prime} \longrightarrow^{*}$ is punctured $l$ times and intersects the boundary of the tubular neighborhood of $K_{2}^{\prime}$ in $l$ parallel copies of the meridian of $K_{2}^{\prime}$. Under the gluing map these meridians match up with $l$ copies of the longitude of $\left(\Sigma_{1}^{\prime}, K_{1}^{\prime}\right)$ on the boundary of the removed tubular neighborhood of $\left(\Sigma_{1}^{\prime}, K_{1}^{\prime}\right)$. So in $\Sigma^{\prime}$ each $l$ times punctured fiber of $\Delta_{2}^{\prime} \longrightarrow^{*}$ is glued along the boundary to $l$ parallel copies of the fiber $G_{1}^{\prime}$ forming a fiber of $K$. Since the fibration of a link in a compact three manifold is unique up to isotopy (see [2, p. 34), this fiber must be diffeomorphic to $G$.

The branch set $G$ can be positioned with respect to the decomposition 3.1 of $F^{\prime}$ so that it lies entirely in $G_{1}^{\prime} \times G_{2}^{\prime} \cup\left(F_{2}^{\prime}\right)^{\circ}$. Then $G$ intersects $G_{1}^{\prime} \times G_{2}^{\prime}$ in $l$ parallel copies of $G_{1}^{\prime}$. The intersection with $\left(F_{2}^{\prime}\right)^{\circ}$ is obtained by pushing the fiber corresponding to $\Delta_{2}^{\prime} \longrightarrow$ into $F_{2}^{\prime}$, so that it is properly embedded and transverse to the properly embedded fiber $G_{2}^{\prime}$, and then removing a tubular neighborhood of this $G_{2}^{\prime}$.

By Theorem 3.2, $F_{1}$ is a branched cyclic cover of $F_{1}^{\prime}$ branched along a properly embedded copy of $G_{1}^{\prime}$. The branch set $G_{1}^{\prime}$ lifts to a properly embedded copy of $G_{1}$ in $F_{1}$. Removing a neighborhood of the properly embedded $G_{1}^{\prime}$ in the base and taking the same cover gives $F_{1}$ minus a neighborhood of the properly embedded $G_{1}$, which is exactly $F_{1}^{\circ}$. Thus, in the branched cyclic cover $(3.2)\left(F_{1}^{\prime}\right)^{\circ}$ lifts to $F_{1}^{\circ}$. Furthermore, the gluing region in the base, $\partial\left(F_{1}^{\prime}\right)^{\circ}=G_{1}^{\prime} \times S^{1}$, lifts to the gluing region in the cover, $G_{1} \times S^{1}$.

By Theorem $3.2, F_{2}$ is the branched cyclic cover of $F_{2}^{\prime}$ branched along a properly embedded version of the fiber of the knot specified by the diagram $\Delta_{2}^{\prime} \longrightarrow{ }^{*}$. Pushing $G_{2}^{\prime}$ into $F_{2}^{\prime}$ so that it is properly embedded and transverse to the properly embedded fiber of $\Delta_{2}^{\prime} \longrightarrow^{*}, G_{2}^{\prime}$ intersects the branch set in $l$ points. On taking the branched cyclic cover, this properly embedded $G_{2}^{\prime}$ lifts to a properly embedded $G_{2}$ in $F_{2}$. If we cross this $G_{2}^{\prime}$ with $G_{1}^{\prime}$ and take the branched cyclic cover branched along the $l$ parallel copies of $G_{1}^{\prime}$ corresponding to the $l$ branch points in $G_{2}^{\prime}$, we obtain $G_{1} \times G_{2}$. Thus, in the branched cyclic cover (3.2) $G_{1}^{\prime} \times G_{2}^{\prime}$ lifts to $G_{1} \times G_{2}$. Now, returning to $F_{2}^{\prime}$ and removing a tubular neighborhood of the properly embedded $G_{2}^{\prime}$ leaves $\left(F_{2}^{\prime}\right)^{\circ}$, and in the cover we have $F_{2}^{\circ}$. Thus, in the branched cyclic cover (3.2) $\left(F_{2}^{\prime}\right)^{\circ}$ lifts to $F_{2}^{\circ}$. So, $F$ decomposes as

$$
F=F_{1}^{\circ} \cup G_{1} \times G_{2} \cup F_{2}^{\circ}
$$

as conjectured.

Remark 3.5. In [1] (see also Remark 8.3 in [18]) Collin and Saveliev remark that their approach to proving the CIC for hypersurfaces $f(x, y)+z^{n}=0$ can be generalized to prove the CIC in the same cases for which Theorem 3.4 proves the MFC. Since the MFC implies the CIC, Theorem 3.4 provides an independent proof of Collin and Saveliev's claim. 
3.2. Equivalent splice diagrams and the Milnor Fiber Conjecture. Recall from section 2.2 that a non-minimal splice diagram is one that fails to satisfy the condition that all edge weights ending in a leaf are strictly greater than 1 . This condition is not necessary in the topological description of homology sphere links specified by splice diagrams, and is only needed to ensure a one-to-one correspondence between diagrams and homology sphere links in Theorem 2.4.

It is often convenient to allow for non-minimal splice diagrams because many more graph knots in homology sphere links can be represented via these splice diagrams. For example, using non-minimal splice diagrams for $S^{3}$, we can represent all algebraic knots in $S^{3}$ (in the sense of links of plane curve singularities as in [2]). In our case the use of non-minimal splice diagrams widens the class of examples to which Theorem 3.4 applies. First, however, we must show that if the MFC holds for a given diagram, then the MFC also holds for any equivalent diagram, assuming that both diagrams satisfy the semigroup condition.

Recall that any non-minimal splice diagram can be reduced to the unique equivalent minimal splice diagram by repeatedly applying the following operations [2]:

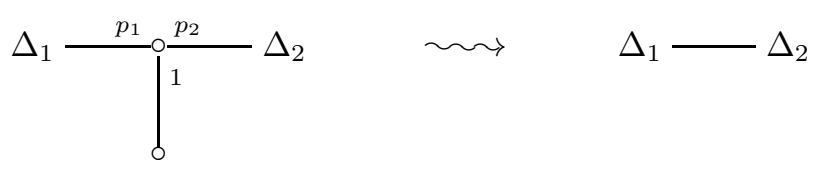

and, for $r>2$,

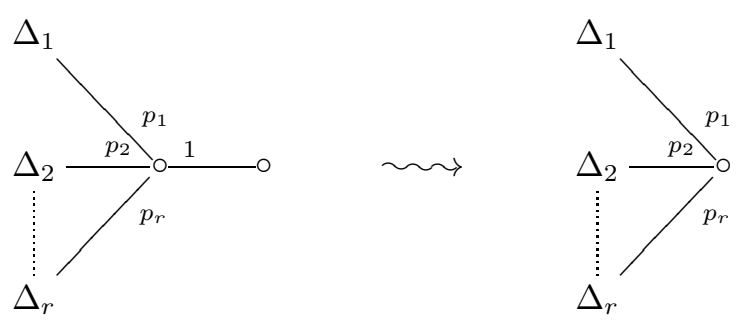

We show that the MFC construction holds for the diagrams on the left if and only if it holds for the diagrams on the right, assuming that both diagrams satisfy the semigroup condition. Thus, if $\Delta$ is a splice diagram satisfying the semigroup condition for a splice type singularity link $\Sigma$ such that the MFC construction holds for $\Delta$, then the MFC holds for any splice diagram for $\Sigma$ that satisfies the semigroup condition.

Lemma 3.6. Let

$$
\Delta=\Delta_{1}-\Delta_{2}
$$

and

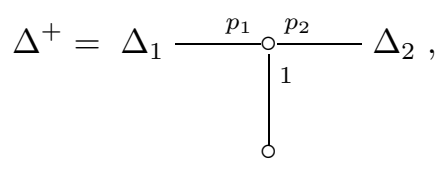

with $r>2$, and suppose that both $\Delta$ and $\Delta^{+}$satisfy the semigroup condition. Then the MFC holds for $\Delta$ if and only if the MFC holds for $\Delta^{+}$.

Proof. Since $\Delta$ and $\Delta^{+}$are equivalent splice diagrams, both of which satisfy the semigroup condition, they correspond to the same splice type singularities with the 
same Milnor fiber, $F$. We first consider the case in which we decompose $\Delta$ along an edge $e$, other than the edge between $\Delta_{1}$ and $\Delta_{2}$, into two subdiagrams, $\Delta_{a}$ and $\Delta_{b}, \Delta=\Delta_{a} \stackrel{e}{-} \Delta_{b}$. Let $F_{a}^{\circ}, F_{b}^{\circ}, G_{a}$ and $G_{b}$, be the pieces in the corresponding MFC construction, where $F_{a}$ is the fiber corresponding to $\Delta_{a}$, and so on. So, conjecturally,

$$
F=F_{a}^{\circ} \cup G_{a} \times G_{b} \cup F_{b}^{\circ} .
$$

Without loss of generality we may assume that $e$ is an edge of $\Delta_{1}$, and that $\Delta_{2}$ is a subdiagram of $\Delta_{b}$. Decompose $\Delta^{+}$along $e$ into subdiagrams $\Delta_{a}^{+}$and $\Delta_{b}^{+}$, and let $\left(F_{a}^{+}\right)^{\circ},\left(F_{b}^{+}\right)^{\circ}, G_{a}^{+}$and $G_{b}^{+}$, be the pieces in the corresponding MFC construction, where $F_{a}^{+}$is the fiber corresponding to $\Delta_{a}^{+}$, and so on. So, the MFC for this decomposition conjectures that

$$
F=\left(F_{a}^{+}\right)^{\circ} \cup G_{a}^{+} \times G_{b}^{+} \cup\left(F_{b}^{+}\right)^{\circ} .
$$

We need to show that (3.3) holds if and only if (3.4) holds. We have $\Delta_{a}=\Delta_{a}^{+}$, so $F_{a}=F_{a}^{+}, G_{a}=G_{a}^{+}$and thus $F_{a}^{\circ}=\left(F_{a}^{+}\right)^{\circ}$. Furthermore, $\Delta_{b}$ and $\Delta_{b}^{+}$are equivalent diagrams, and the knots along which the splice decomposition occurs are the same, so $F_{b}=F_{b}^{+}, G_{a}=G_{b}^{+}$and thus $F_{b}^{\circ}=\left(F_{b}^{+}\right)^{\circ}$. So,

$$
F_{a}^{\circ} \cup G_{a} \times G_{b} \cup F_{b}^{\circ}=\left(F_{a}^{+}\right)^{\circ} \cup G_{a}^{+} \times G_{b}^{+} \cup\left(F_{b}^{+}\right)^{\circ} .
$$

Thus, the MFC construction (3.3) corresponding to decomposing $\Delta$ along $e$ yields $F$ if and only if the MFC construction (3.4) corresponding to decomposing $\Delta^{+}$ along $e$ yields $F$.

Now, the node in $\Delta^{+}$not contained in either $\Delta_{1}$ or $\Delta_{2}$ we will call $v$ :

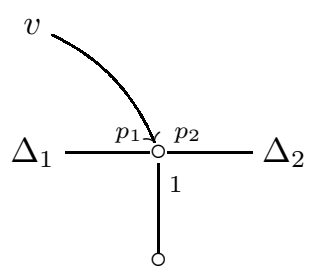

We now consider the case in which we decompose $\Delta^{+}$along an edge $e$ which is not an edge of $\Delta_{1}$ or $\Delta_{2}$. Then there are three sub-cases:

(1) $e$ is the edge from $v$ to a leaf:

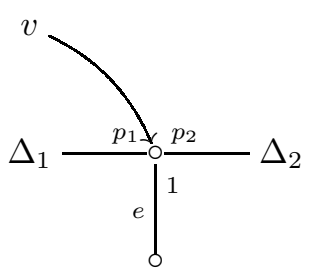


(2) $e$ is the edge from $\Delta_{1}$ to $v$ :

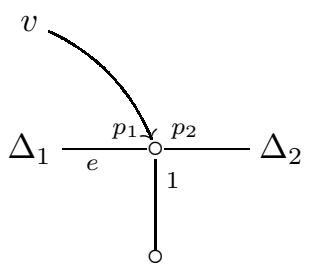

(3) $e$ is the edge from $\Delta_{2}$ to $v$ :

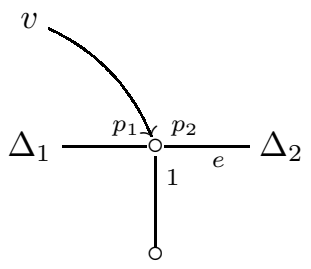

The MFC corresponding to the decomposition of case (1) is always true. The MFC corresponding to the decomposition in case (2) is true if and only if the MFC corresponding to the decomposition in case (3) is true. Consider case (2). We will show that the MFC corresponding to this decomposition of $\Delta^{+}$holds if and only if the MFC holds when we decompose $\Delta$ along the edge from $\Delta_{1}$ to $\Delta_{2}$.

Let $\Delta_{a}^{+}$be the subdiagram in this decomposition containing $\Delta_{1}$ and let $\Delta_{b}^{+}$be the subdiagram in this decomposition containing $\Delta_{2}$. Let $\left(F_{a}^{+}\right)^{\circ},\left(F_{b}^{+}\right)^{\circ}, G_{a}^{+}$and $G_{b}^{+}$be the pieces in the corresponding MFC construction. Decompose $\Delta$ along the edge between $\Delta_{1}$ and $\Delta_{2}$. Let $\Delta_{a}$ be the piece of this decomposition consisting of $\Delta_{1}$ and let $\Delta_{b}$ be the piece of this decomposition consisting of $\Delta_{2}$. Let $F_{a}^{\circ}, F_{b}^{\circ}, G_{a}$ and $G_{b}$ be the pieces in the corresponding MFC construction as before. Then $\Delta_{a}=\Delta_{a}^{+}$. So, $F_{a}=F_{a}^{+}$and $G_{a}=G_{a}^{+}$, and thus $F_{a}^{\circ}=\left(F_{a}^{+}\right)^{\circ}$. On the other side, $\Delta_{b}$ is equivalent to $\Delta_{b}^{+}$, so $F_{b}=F_{b}^{+} . G_{b}^{+}$is the fiber of the knot indicated by

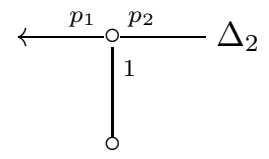

while $G_{b}$ is the fiber of the knot indicated by

$$
\longleftarrow \Delta_{2}
$$

From the splice diagram we can see that $G_{b}^{+}$can be built by gluing a once punctured fiber of the knot indicated by

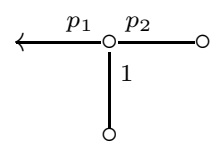

to the fiber $G_{b}$ along the boundary. The knot indicated by

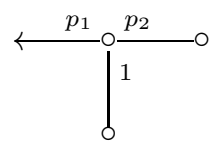


is an unknotted circle in $S^{3}$ and so has fiber $D^{2}$. Thus $G_{b}^{+}$is the result of pasting an annulus along one boundary component to the boundary of $G_{b}$, giving back $G_{b}$. So,

$$
F_{a}^{\circ} \cup G_{a} \times G_{b} \cup F_{b}^{\circ}=\left(F_{a}^{+}\right)^{\circ} \cup G_{a}^{+} \times G_{b}^{+} \cup\left(F_{b}^{+}\right)^{\circ} .
$$

Thus, the MFC construction corresponding to decomposing $\Delta$ along the edge between $\Delta_{1}$ and $\Delta_{2}$ yields $F$ if and only if the MFC construction corresponding to decomposing $\Delta^{+}$along $e$ yields $F$.

Lemma 3.7. Let

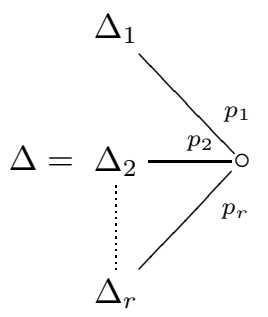

and

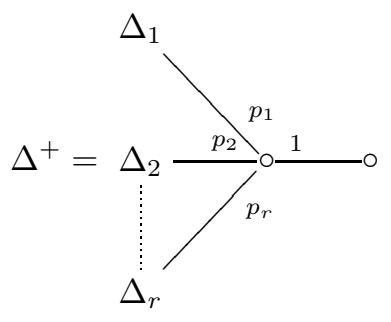

and suppose that both $\Delta$ and $\Delta^{+}$satisfy the semigroup condition. Then the MFC holds for $\Delta$ if and only if the MFC holds for $\Delta^{+}$.

Proof. Since both $\Delta$ and $\Delta^{+}$are equivalent splice diagrams satisfying the semigroup condition, they correspond to the same splice type singularities with the same Milnor fiber, $F$. When we decompose $\Delta$ along an edge, decomposing $\Delta^{+}$along the corresponding edge leads to equivalent splice components and all pieces in the MFC construction are the same. Thus, the MFC holds for decompositions of $\Delta$ along an edge if and only if the MFC construction holds for the decomposition of $\Delta^{+}$along the same edge. If we decompose $\Delta^{+}$along the edge not contained in $\Delta$,

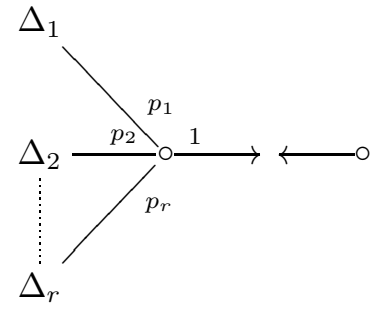


Then the MFC construction amounts to removing a tubular neighborhood of the knot

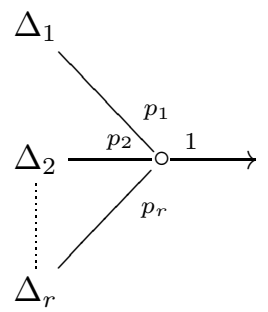

from $F$, gluing it back in and then pasting a collar neighborhood on to the boundary, giving back $F$.

Since any splice diagram can be reduced to the unique equivalent minimal splice diagram via these operations, we have proven:

Theorem 3.8. If the Milnor Fiber Conjecture holds for a given diagram, then the Milnor Fiber Conjecture also holds for any equivalent diagram.

3.3. An example. In light of Theorem 3.8 it is not hard to write examples to which Theorem 3.4 applies.

To take a concrete example, start with the splice type singularity specified by the diagram

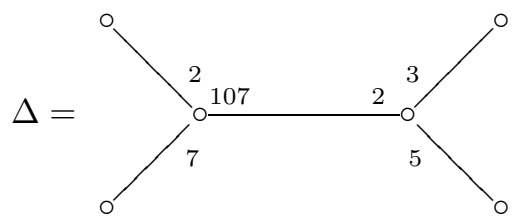

Label the leaves $w_{1}, \ldots, w_{4}$ starting at the upper left leaf and moving counterclockwise, and associate a variable $z_{i}$ to $w_{i}$. Call the left node $v_{1}$ and the right node $v_{2}$.

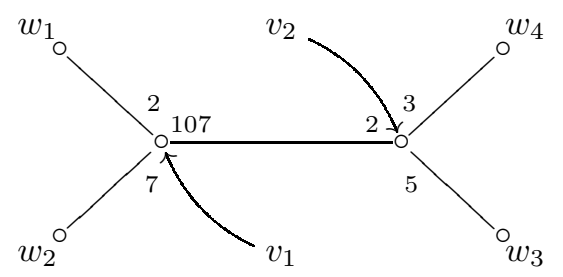

Then

\begin{tabular}{|r|r|r|}
\hline$i$ & $l_{v_{1} w_{i}}^{\prime}$ & $l_{v_{2} w_{i}}^{\prime}$ \\
\hline 1 & 1 & 7 \\
\hline 2 & 1 & 2 \\
\hline 3 & 3 & 1 \\
\hline 4 & 5 & 1 \\
\hline
\end{tabular}

The semigroup condition at the left node is

$$
\begin{aligned}
2 & =\alpha_{v_{1} w_{1}} \cdot 1 \\
7 & =\alpha_{v_{1} w_{2}} \cdot 1 \\
107 & =\alpha_{v_{1} w_{3}} \cdot 3+\alpha_{v_{1} w_{4}} \cdot 5,
\end{aligned}
$$


and at the right node is

$$
\begin{aligned}
& 2=\alpha_{v_{2} w_{1}} \cdot 7+\alpha_{v_{2} w_{2}} \cdot 2, \\
& 5=\alpha_{v_{2} w_{3}} \cdot 1 \\
& 3=\alpha_{v_{2} w_{4}} \cdot 1
\end{aligned}
$$

The condition is satisfied by any choice of coefficients

\begin{tabular}{|r|r|r|}
\hline$i$ & $\alpha_{v_{1} w_{i}}$ & $\alpha_{v_{2} w_{i}}$ \\
\hline 1 & 2 & 0 \\
\hline 2 & 7 & 1 \\
\hline 3 & $4+j \cdot 5$ & 5 \\
\hline 4 & $19-j \cdot 3$ & 3 \\
\hline
\end{tabular}

where $j \in\{0, \ldots, 6\}$. So, for example using the coefficients with $j=1$, the splice type equations can be chosen to be

$$
\begin{aligned}
z_{1}^{2}+z_{2}^{7}+z_{3}^{9} z_{4}^{16} & =0 \\
-z_{2}+z_{3}^{5}+z_{4}^{3} & =0 .
\end{aligned}
$$

Solving for $z_{2}$ in the second equation and substituting into the first, we obtain

$$
z_{1}^{2}+\left(z_{3}^{5}+z_{4}^{3}\right)^{7}+z_{3}^{9} z_{4}^{16}=0 .
$$

Changing variables to $x=z_{3}, y=z_{4}$ and $z=z_{1}$ gives

$$
\left(x^{5}+y^{3}\right)^{7}+x^{9} y^{16}+z^{2}=0 .
$$

Letting $f_{1}(x, y)=\left(x^{5}+y^{3}\right)^{7}+x^{9} y^{16}$, this is

$$
f_{1}(x, y)+z^{2}=0 .
$$

Thus, the MFC is known to hold for this splice type singularity by [18]. Now, take an equivalent non-minimal version of this diagram that still satisfies the semigroup condition, such as,

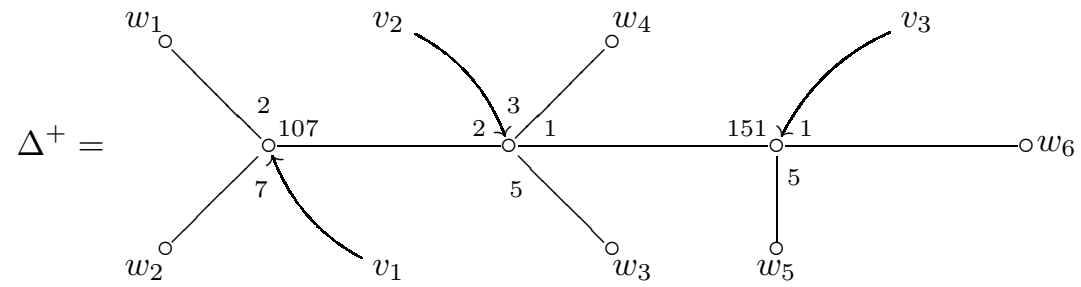

Label the leaves and nodes as shown, and associate a variable $z_{i}$ to the leaf $w_{i}$. Then

\begin{tabular}{|r|r|r|r|}
\hline$i$ & $l_{v_{1} w_{i}}^{\prime}$ & $l_{v_{2} w_{i}}^{\prime}$ & $l_{v_{3} w_{i}}^{\prime}$ \\
\hline 1 & 1 & 7 & 105 \\
\hline 2 & 1 & 2 & 30 \\
\hline 3 & 3 & 1 & 6 \\
\hline 4 & 5 & 1 & 10 \\
\hline 5 & 15 & 1 & 1 \\
\hline 6 & 17 & 5 & 1 \\
\hline
\end{tabular}


One choice of coefficients that satisfies the semigroup condition is

\begin{tabular}{|r|r|r|r|}
\hline$i$ & $\alpha_{v_{1} w_{i}}$ & $\alpha_{v_{2} w_{i}}$ & $\alpha_{v_{3} w_{i}}$ \\
\hline 1 & 2 & 0 & 1 \\
\hline 2 & 7 & 2 & 1 \\
\hline 3 & 9 & 5 & 1 \\
\hline 4 & 16 & 3 & 1 \\
\hline 5 & 0 & 1 & 5 \\
\hline 6 & 0 & 0 & 1 \\
\hline
\end{tabular}

For this choice of coefficients, the corresponding admissible monomials are $z_{1}^{2}$, $z_{2}^{7}$ and $z_{3}^{9} z_{4}^{16}$ at $v_{1} ; z_{2}, z_{3}^{5}, z_{4}^{3}$ and $z_{5}$ at $v_{2}$; and $z_{1} z_{2} z_{3} z_{4}, z_{5}^{5}$ and $z_{6}$ at $v_{3}$. Thus, splice type equations can be chosen to be

$$
\begin{aligned}
z_{1}^{2}+z_{2}^{7}+z_{3}^{9} z_{4}^{16}=0 \\
-z_{2}+z_{3}^{5}+z_{4}^{3}=0, \\
z_{3}^{5}+2 z_{4}^{3}-z_{5}=0, \\
z_{1} z_{2} z_{3} z_{4}+z_{5}^{5}-z_{6}=0 .
\end{aligned}
$$

Substituting for $z_{2}$ and changing variables to $x=z_{3}, y=z_{4}, z=z_{1}, Z_{5}=$ $z_{5}-\left(z_{3}^{5}+2 z_{4}^{3}\right)$ and $Z_{6}=z_{6}-\left(z_{1} z_{2} z_{3} z_{4}+z_{5}^{5}\right)$, we have

$$
\begin{aligned}
\left(x^{5}+y^{3}\right)^{7}+x^{9} y^{16}+z^{2} & =0, \\
Z_{5} & =0, \\
Z_{6} & =0,
\end{aligned}
$$

the same splice type singularity as before. By applying Theorem 3.8, the MFC is also valid for splice decompositions of this diagram.

Now, consider the graph knot indicated by the right-most leaf:

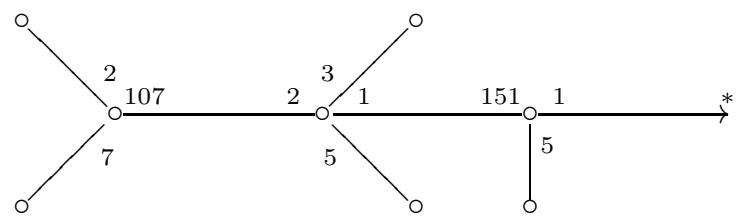

By Theorem 2.5 this knot is cut out by setting

$$
z_{6}=z_{1} z_{2} z_{3} z_{4}+z_{5}^{5}=0
$$

in the original variables of equation (3.5). Substituting for $z_{2}$ and $z_{5}$ from (3.6), this becomes

$$
z_{1} z_{3} z_{4}\left(z_{3}^{5}+z_{4}^{3}\right)+\left(z_{3}^{5}+2 z_{4}^{3}\right)^{5}=0 .
$$

So, in the variables $x, y, z$, the knot is cut out by

$$
f_{2}(x, y, z):=x y z\left(x^{5}+y^{3}\right)+\left(x^{5}+2 y^{3}\right)^{5}=0 .
$$

Since 11 is coprime to all edge weights near with respect to $*$, the diagram

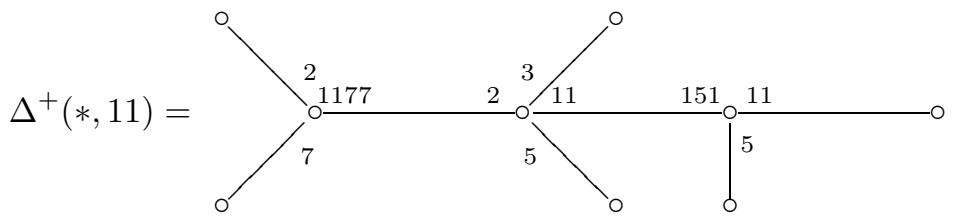


is again a splice diagram for a homology sphere link by Theorem 3.1. By Lemma 3.3. the splice type equations for this singularity can be obtained by replacing $z_{6}$ with $z_{6}^{11}$ in the equations (3.6), yielding,

$$
\begin{aligned}
z_{1}^{2}+z_{2}^{7}+z_{3}^{9} z_{4}^{16}=0 \\
-z_{2}+z_{3}^{5}+z_{4}^{3}=0 \\
z_{3}^{5}+2 z_{4}^{3}-z_{5}=0 \\
z_{1} z_{2} z_{3} z_{4}+z_{5}^{5}-z_{6}^{11}=0 .
\end{aligned}
$$

Substituting for $z_{2}$ and $z_{5}$ and changing variables to $x=z_{3}, y=z_{4}, z=z_{1}, w=z_{6}$ and $Z_{5}=z_{5}-\left(z_{3}^{5}+2 z_{4}^{3}\right)$, splice type equations for $\Delta^{+}(*, 11)$ are

$$
\begin{aligned}
\left(x^{5}+y^{3}\right)^{7}+x^{9} y^{16}+z^{2} & =0, \\
\left(x^{5}+2 y^{3}\right)^{5}+x y\left(x^{5}+y^{3}\right) z+w^{11} & =0 .
\end{aligned}
$$

With $f_{1}(x, y)$ and $f_{2}(x, y, z)$ as before, this is

$$
\begin{aligned}
f_{1}(x, y)+z^{2} & =0, \\
f_{2}(x, y, z)+w^{11} & =0 .
\end{aligned}
$$

By Theorem 3.4 the MFC is valid for this singularity.

3.4. Iterated branched cyclic covers and the Splice Type Conjecture. One may notice that in the example of section 3.3 the equations for $\Delta^{+}(*, k)$ were the same as those for $\Delta$, with an additional equation added of the form $f_{2}(x, y, z)+w^{k}=$ 0 , where $f_{2}(x, y, z)=0$ is the equation that cut out the graph knot along which the branching occurs. This is true in general. The splice type equations for a singularity obtained by the construction in section 3.1 are of the form $\left\{f_{i}\left(z_{1}, \ldots, z_{i+1}\right)+z_{i+2}^{k_{i}}=\right.$ $0\}_{i=1}^{n}$, and one is tempted to try and use Theorem 3.4 to prove the MFC for all isolated complete intersection singularities with homology sphere link given by equations of this form. The problem is that, without a proof of the Splice Type Conjecture, we cannot say that such a complete intersection is necessarily of splice type.

Implicit in Neumann and Wahl's statement that the MFC holds for hypersurface singularities of the form $f(x, y)+z^{k}=0$ is the assertion that such a singularity is of splice type, since the MFC only applies in the case of splice type singularities. The proof that a hypersurface of the form $f(x, y)+z^{k}=0$ with homology sphere link is of splice type is a straightforward consequence of the positive edge determinant condition and Lemma 2.6 (see proposition 8.1 of [18]). This argument does not generalize to equations of the form $\left\{f_{i}\left(z_{1}, \ldots, z_{i+1}\right)+z_{i+2}^{k_{i}}=0\right\}_{i=1}^{n}$. In fact, a minimal splice diagram for the link of a splice type singularity given by equations of the form $\left\{f_{i}\left(z_{1}, \ldots, z_{i+1}\right)+z_{i+2}^{k_{i}}=0\right\}_{i=1}^{n}$ may be equivalent to a non-minimal diagram that does not satisfy the semigroup condition.

For example, the link $\Sigma$ of the hypersurface singularity from the example in section 3.3 given by

$$
\left(x^{5}+y^{3}\right)^{7}+x^{9} y^{16}+z^{2}=0
$$


is specified by the diagram

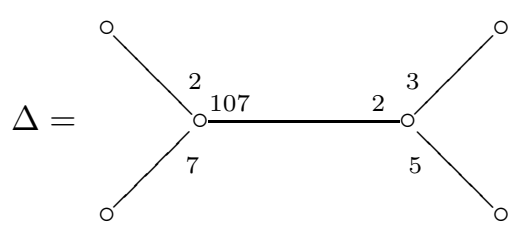

and this diagram satisfies the semigroup condition. Now consider the equivalent non-minimal diagram

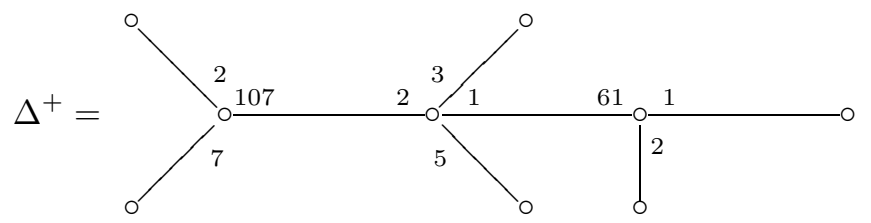

This splice diagram does not satisfy the semigroup condition, even though it corresponds to the link of a splice type singularity. If the STC is true, then the knot indicated by the right-most vertex cannot be cut out by a single equation. If the knot indicated by the right-most vertex were cut out by a single equation, $f(x, y, z)=0$, then for any $k$ pairwise coprime to $2,3,5,7$ and 61 , the diagram,

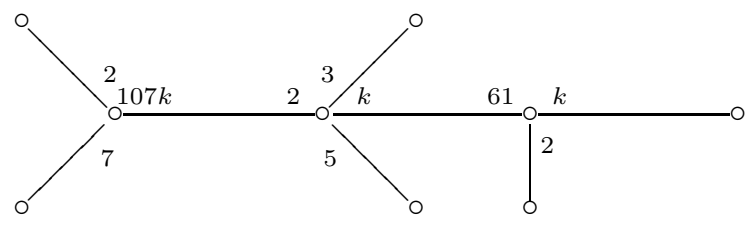

would correspond to a complete intersection given by the equations

$$
\begin{gathered}
\left(x^{5}+y^{3}\right)^{7}+x^{9} y^{16}+z^{2}=0, \\
f(x, y, z)+w^{k}=0,
\end{gathered}
$$

but not of splice type, and would thus be a counterexample to the STC.

The question then becomes, when is the knot indicated by a distinguished leaf of a splice diagram a graph knot? This seems to be a particularly tricky question. Through the use of Lemma 2.6 one can get bounds on certain coefficients in the splice diagrams that force the semigroup condition to hold, but as the example above illustrates, there is no a priori reason for these bounds to be satisfied. Pursuit of an answer to this question, either providing a counterexample to the STC or a proof of the STC for the singularities considered in this paper is an interesting direction for further research.

\section{REFERENCES}

1. Olivier Collin and Nikolai Saveliev, Equivariant Casson invariants via gauge theory, J. Reine Agnew. Math. 541 (2001), 143-169. MR1876288(2002k:57077)

2. David Eisenbud and Walter Neumann, Three-dimensional link theory and invariants of plane curve singularities, Annals of Mathematics Studies, vol. 110, Princeton University Press, Princeton, 1985. MR817982 (87g:57007)

3. R. Fintushel and R. Stern, Instanton homology groups of Seifert fibered homology three spheres, Proc. London Math. Soc. 61 (1990), 109-137. MR.1051101(91k:57029) 
4. Helmut Hamm, Lokale topologische Eigenschaften komplexer Ráume, Math. Ann. 191 (1971), 235-252. MR0286143 (44:3357)

5. _— Exotische Sphären als Umgebungsränder in speziellen komplexen Räuman, Math. Ann. 197 (1972), 44-56. MR0314073 (47:2625)

6. Louis Kauffman and Walter Neumann, Products of knots, branched fibrations, and sums of singularities, Topology 16 (1977), 369-393. MR0488073 (58:7644)

7. E. J. N. Looijenga, Isolated singular points on complete intersections, London Mathematical Society Lecture Note Series, vol. 77, Cambridge University Press, Cambridge, 1984. MR.747303 (86a:32021)

8. I. Luengo-Velasco, A. Melle-Hernández, and A. Némethi, Links and analytic invariants of superisolated singularities, J. Algebraic Geom. 14 (2005), 543-565. MR2129010 (2005m:32057)

9. John Milnor, Singular points of complex hypersurfaces, Annals of Mathematics Studies, vol. 61, Princeton University Press, Princeton, 1968. MR0239612 (39:969)

10. András Némethi, Invariants of normal surface singularities, Real and complex singularities, Contemp. Math., vol. 354, Amer. Math. Soc., Providence, RI, 2004, pp. 161-208. MR2087811 (2005g:32040)

11. András Némethi and Liviu I. Nicolaescu, Seiberg-Witten invariants and surface singularities III: Splicings and cyclic covers.

12. _ Seiberg-Witten invariants and surface singularities, Geom. Topol. 6 (2002), 269-328. MR:1914570 (2003i:14048)

13. - Seiberg-Witten invariants and surface singularities II: Singularities with good $\mathbb{C}^{*}$ action, J. London Math. Soc. 69 (2004), 593-607. MR.2050035 (2005g:14070)

14. Walter D. Neumann, Abelian covers of quasihomogeneous surface singularities, Singularities, Part 2 (Arcata, Calif., 1981), Proc. Sympos. Pure Math., vol. 40, Amer. Math. Soc., Providence, RI, 1983, pp. 233-243. MR713252 (85g:32018)

15. Walter D. Neumann and Frank Raymond, Seifert manifolds, plumbing, $\mu$-invariant and orientation reversing maps, Algebraic and geometric topology (Proc. Sympos., Univ. California, Santa Barbara, Calif., 1977), Lecture Notes in Math., vol. 664, Springer, Berlin, 1978, pp. 163196. MR518415 (80e:57008)

16. Walter D. Neumann and Jonathan Wahl, Casson invariant of links of singularities, Comment. Math. Helv. 65 (1990), no. 1, 58-78. MR.1036128 (91c:57022)

17. Universal abelian covers of quotient-cusps, Math. Ann. 326 (2003), no. 1, 75-93. MR 1981612 (2004d:32039)

18. Complete intersection singularities of splice type as universal abelian covers, Geom. Topol. 9 (2005), 699-755 (electronic). MR2140991

19. _ Complex surface singularities with integral homology sphere links, Geom. Topol. 9 (2005), 757-811 (electronic). MR:2140992 (2006b:32042)

20. Tomohiro Okuma, Universal abelian covers of certain surface singularities.

Department of Mathematics, Columbia University, New York, New York 10027

Current address: Center for the Study of Complex Systems, University of Michigan, Ann Arbor, Michigan 48109

E-mail address: pjlamber@umich.edu 